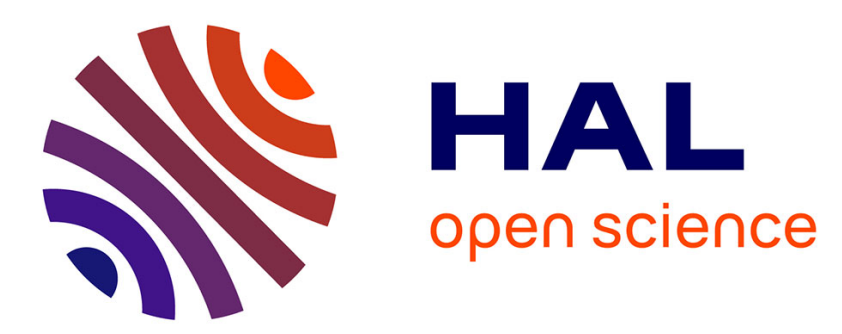

\title{
Adaptive mesh refinements for thin shells whose middle surface is not exactly known
}

Philippe Destuynder, Yann Moguen, Michel Salaün

\section{To cite this version:}

Philippe Destuynder, Yann Moguen, Michel Salaün. Adaptive mesh refinements for thin shells whose middle surface is not exactly known. Computer Methods in Applied Mechanics and Engineering, 2008, 10.1016/j.cma.2008.07.001 . hal-02358810

\section{HAL Id: hal-02358810 https://hal-univ-pau.archives-ouvertes.fr/hal-02358810}

Submitted on 13 Nov 2019

HAL is a multi-disciplinary open access archive for the deposit and dissemination of scientific research documents, whether they are published or not. The documents may come from teaching and research institutions in France or abroad, or from public or private research centers.
L'archive ouverte pluridisciplinaire HAL, est destinée au dépôt et à la diffusion de documents scientifiques de niveau recherche, publiés ou non, émanant des établissements d'enseignement et de recherche français ou étrangers, des laboratoires publics ou privés. 
1

\title{
Adaptive mesh refinements for thin shells the middle surface of which is not known
}

\author{
Ph. Destuynder ${ }^{a}$, Y. Moguen ${ }^{\text {b,*, M. Salaün }}{ }^{\mathrm{c}}$ \\ ${ }^{a}$ Conservatoire National des Arts et Métiers - Chaire de Calcul Scientifique \\ 292 rue Saint-Martin, F-75141 Paris cedex 03, France. \\ ${ }^{\mathrm{b}}$ Université de Pau et des Pays de l'Adour \\ Laboratoire de Thermique, Energétique et Procédés - IUT GTE \\ 1 avenue de l'Université, F-64000 Pau, France. \\ ${ }^{\mathrm{c}}$ Université de Toulouse - ISAE - DMSM \\ 10 avenue E. Belin, F-31055 Toulouse, France.
}

\begin{abstract}
A strategy concerning mesh refinements for thin shells computation is presented. The geometry of the shell is given only by the reduced information consisting in nodes and normals on its middle surface corresponding to a coarse mesh. The new point is that the mesh refinements are defined from several criteria, including the transverse shear stress which doesn't appear in the mechanical energy, and enables one to construct the unknown middle surface step by step. In fact, it can be interpreted as an optimum design algorithm where the control variable is the mapping which defines the middle surface.
\end{abstract}

Key words: Shell, adaptive mesh refinement, geometry approximation, mixed finite elements method, a posteriori error estimation 


\section{Introduction}

- The problem of adaptive mesh refinements on shells remains a seldom studied subject. Compared with more classical models like plates or bidimensional elasticity problems, one has to deal with an additional difficulty which is that the mesh to refine is not plane. For instance, the center of mass of a triangle or of a quadrangle is generally not on the middle surface of the shell even if the nodes are on it. It is the reason why it is often assumed that the exact shell geometry is known, analytically or from a computer aided design program. For example, in [1], [2], [3] and [4], the exact mapping defining the shell geometry is supposed to be known and a completely new mesh, based on spatial distribution of new element sizes, is defined at each step of the refinement procedure. In another way, it is suggested in [5] to create a new mesh by moving the vertices of the previous one, while isogeometric formulations used in [6], assumes that the geometry is exactly known from NURBS. This enables one to construct a mesh of "NURBS elements", which is easy to refine by reindexation of the parametric space. Despite their interest, these two approaches also need the knowledge of the exact geometry of the shell surface. Finally, in the case of shell structures, [7] is one of the very few papers in which the initial mesh is the basis of the next one, in the sense that some of its elements are divided in order to obtain the new mesh. But again, these authors assume that the exact shell geometry is known. In fact, they introduce in their numerical experiments the idea of quality of the geometry approximation as a refinement criteria. This one is expressed as a function of the errors on the added nodes position and the unit normal vectors at these points.

\footnotetext{
* Corresponding author. Tel.: +335594071 59; fax: +33559407160.

Email address: yann.moguen@free.fr (Y. Moguen).
} 
- In this paper, a new approach is suggested. A refinement strategy is introduced in association with an approximation of the middle surface of the shell. It is only assumed that (1) the positions of the vertices of a set of flat triangular elements approximating the middle surface and (2) the normal vectors at these vertices, are known from a coarse mesh of the middle surface of the shell. Then, following a methodology developed in [8], it is possible to build new vertices for each element which are closer to the middle surface than the middle of the element edges.

- Concerning the choice of the refinement criteria, let us make few remarks. The usual estimators, such as those of Zhu and Zienkiewicz (see [9] and [10]) for example, are appropriate for a global error control. Nevertheless, in some papers (see for exemple [11] and [12]), the concept of variable of interest is introduced in the definition of the error indicator. In the case of thin structures, such as plates and shells, an obvious quantity of interest is the transverse shear stress. By the way, among Kirchhoff-Love assumptions, one is the nullity of the transverse shear strain (but not the shear stress). Moreover, the transverse shear stress is considered as neglectible in front of the inplane stresses in the constitutive relationship. But, it is precisely the stress component which is necessary in order to satisfy the three-dimensional equilibrium equation through the thickness of the shell. So, it can be pointwise very different from zero (it can be a mathematical measure). Obviously, this phenomenon should be taken into account in the refinement criteria. It is the basic point in the definition of the error indicator described herafter.

- The plan of this paper is the following one. In section 2, few notations related to shells are introduced. The finite element formulation used is described in section 3, while section 4 concerns the mesh refinement strategy. In section 
5 , one introduces the mesh refinement criteria and, finally, various numerical examples are presented in the last section.

\section{Geometrical aspects for shells}

- Let us recall that a shell is a three-dimensional structure for which one dimension, called the thickness, is very small compared to the other dimensions (maximum length or local radius of curvature). In this paper, the middle surface of the shell will be denoted by $\omega$ while half the thickness will be $\varepsilon$. Finally, as a shell formulation is used, even if it is a mixed one, let us introduce now some basic elements of differential geometry. For more details see [13], for example.

- So, it is assumed that there exists a mapping, say $\phi$, from an open set $\widehat{\omega}$ onto the middle surface $\omega$ which is at least $\mathcal{C}^{3}(\widehat{\omega})$. The plane $\mathbb{R}^{2}$ containing $\widehat{\omega}$ will be referred to coordinates $\left(\xi^{1}, \xi^{2}\right)$ while the space $\mathbb{R}^{3}$ is referred to an orthonormal system of coordinates $\left(O ; e_{1}, e_{2}, e_{3}\right)$. Then, one has

$$
\omega=\left\{m \in \mathbb{R}^{3} \mid m=\phi\left(\xi^{1}, \xi^{2}\right),\left(\xi^{1}, \xi^{2}\right) \in \widehat{\omega}\right\} .
$$

Corresponding to the mapping $\phi$, a curvilinear system of coordinates is defined on $\omega$ such that, at any point $m=\phi\left(\xi^{1}, \xi^{2}\right)$ of $\omega$, the tangent vectors are (see Figure 1):

$$
a_{1}=\phi_{, 1} \equiv \frac{\partial \phi}{\partial \xi^{1}} \quad, \quad a_{2}=\phi_{, 2} \equiv \frac{\partial \phi}{\partial \xi^{2}}
$$

Let us assume that the vectors $a_{1}$ and $a_{2}$ are linearly independent and span the tangent plane at each point $m$ of $\omega$. The unit normal vector at point $m$ is 
then defined by

$$
N=\frac{a_{1} \wedge a_{2}}{\left\|a_{1} \wedge a_{2}\right\|}
$$

For the sake of brevity, in the following, Greek indices are assumed to belong to the set $\{1,2\}$ and the implicit summation convention over repeated indices is adopted.

- Let us now define several quantities, which are necessary for the definition of the shell model. The first fundamental form on surface $\omega$ is given by

$$
g_{\alpha \beta}=a_{\alpha} \cdot a_{\beta}
$$

where "." stands for the euclidian scalar product. This tensor is also called the metric tensor. Its determinant is $|g|=g_{11} g_{22}-g_{12}^{2}$ and its inverse is such that:

$$
g^{\alpha \beta}=a^{\alpha} \cdot a^{\beta},
$$

where $\left\{a^{\alpha}\right\}$, called the dual basis of $\left\{a_{\alpha}\right\}$, is defined with the vectors $a^{\alpha}$ such that: $a^{\alpha} \cdot a_{\beta}=\delta_{\beta}^{\alpha}$ (Kronecker's symbol). Then, let us introduce $a_{\alpha, \beta}$, which is the partial derivative of $a_{\alpha}$ with respect to $\xi^{\beta}$. One can write this vector in the basis $\left(a_{1}, a_{2}, N\right)$ and obtain $a_{\alpha, \beta}=\Gamma_{\alpha \beta}^{\gamma} a_{\gamma}+b_{\alpha \beta} N$, where $\Gamma_{\alpha \beta}^{\gamma}$ are the Christoffel's symbols. $b_{\alpha \beta}$ is the second fundamental form on $\omega$, also called the curvature. One has

$$
b_{\alpha \beta}=N \cdot a_{\alpha, \beta} \quad, \quad \Gamma_{\alpha \beta}^{\gamma}=a^{\gamma} \cdot a_{\alpha, \beta} .
$$




\section{A mixed variational formulation for the Koiter's shell model}

\subsection{The Koiter's shell model}

- The Koiter's shell model is used (see [14] and [15]). It is formulated using the two following strain tensors. The first one represents the change of metric on the surface $\omega$ due to a displacement. It is denoted by $\gamma_{\alpha \beta}$. The second one, named $\rho_{\alpha \beta}$, is the change of curvature suggested by Budiansky and Sanders [15]. If $v$ is a displacement vector field on the surface $\omega$, expressed in local coordinates by $v=v^{\alpha} a_{\alpha}+v_{3} N$, then $\gamma_{\alpha \beta}(v)$ and $\rho_{\alpha \beta}(v)$ are respectively given by

$$
\left\{\begin{array}{l}
\gamma_{\alpha \beta}(v)=\frac{1}{2}\left(v_{\alpha \mid \beta}+v_{\beta \mid \alpha}\right)-b_{\alpha \beta} v_{3} \\
\rho_{\alpha \beta}(v)=\frac{1}{2}\left(\theta_{\alpha \mid \beta}+\theta_{\beta \mid \alpha}\right)+\frac{1}{2}\left(b_{\alpha}^{\lambda} v_{\beta \mid \lambda}+b_{\beta}^{\lambda} v_{\alpha \mid \lambda}\right)-b_{\alpha}^{\lambda} b_{\lambda \beta} v_{3}
\end{array},\right.
$$

with $b_{\alpha}^{\lambda}=g^{\lambda \beta} b_{\beta \alpha}$ and $v_{\alpha \mid \beta}=v_{\alpha, \beta}-\Gamma_{\alpha \beta}^{\gamma} v_{\lambda}$ (covariant derivative). Finally, the transverse section rotation $\theta$ can be expressed through the Kirchhoff-Love kinematical relation by

$$
\theta_{\alpha}=-b_{\alpha}^{\lambda} v_{\lambda}-v_{3, \alpha}
$$

An important point to notice is the following one. The expression of $\rho_{\alpha \beta}(v)$ requires the derivatives of $\theta_{\alpha}$ so of $b_{\alpha}^{\lambda}$. As $b_{\alpha}^{\lambda}$ depends on the second derivatives of the mapping $\phi$ (see (3)), such a model needs the third derivatives of $\phi$.

- Hence, the classical shell model consists in finding a displacement field $u$ belonging to the set $V$ of the admissible displacement fields, and such that for 
all $v \in V$,

$$
\int_{\omega} R^{M \alpha \beta \lambda \mu} \gamma_{\alpha \beta}(u) \gamma_{\lambda \mu}(v)+\frac{\varepsilon^{2}}{3} \int_{\omega} R^{F \alpha \beta \lambda \mu} \rho_{\alpha \beta}(u) \rho_{\lambda \mu}(v)=l(v)
$$

where $R^{M \alpha \beta \lambda \mu}$ and $R^{F \alpha \beta \lambda \mu}$ are respectively the membrane and the bending stiffness tensors. In the particular case of an homogeneous and isotropic material,

$R^{M \alpha \beta \lambda \mu}=R^{F \alpha \beta \lambda \mu}=\frac{E \varepsilon}{1-\nu^{2}}\left((1-\nu)\left(g^{\alpha \lambda} g^{\beta \mu}+g^{\alpha \mu} g^{\beta \lambda}\right)+2 \nu g^{\lambda \mu} g^{\alpha \beta}\right)$

where $E$ is the Young's modulus and $\nu$ the Poisson's ratio. Finally, $l(v)$ stands for the mechanical loading.

\subsection{Continuous mixed variational formulation}

- The main ideas of the mixed formulation, introduced in [16], are the following. First of all, the transverse section rotation $\theta$ is introduced as a new unknown. This is very usual in plate and shell theories. Second, the KirchhoffLove relationship (5) is prescribed by means of a Lagrange multiplier. Let us observe that this multiplier has the physical meaning of the resultant transverse shear stress, which will be useful for the definition of refinement criteria.

- Let us now be more precise. We introduce the membrane stress tensor $n^{\alpha \beta}=R^{M \alpha \beta \lambda \mu} \gamma_{\lambda \mu}(u)$, the bending moment $m^{\alpha \beta}=R^{F \alpha \beta \lambda \mu} \rho_{\lambda \mu}(u)$, where $\gamma_{\lambda \mu}(u)$ and $\rho_{\lambda \mu}(u)$ are given in (4), and the resultant transverse shear stress, say $q=q^{\alpha} a_{\alpha}$. The basic point of the method is to split $q$ into the sum of the gradient of a scalar function and the rotational of another one (Helmholtz decomposition), like 


$$
q=\operatorname{grad} \varphi+\operatorname{rot} \psi=g^{\alpha \beta} \varphi_{, \beta} a_{\alpha}+\frac{1}{\sqrt{|g|}}\left(-\psi_{, 2} a_{1}+\psi_{, 1} a_{2}\right)
$$

So, starting from the equilibrium relationships and writing (7) in a variational form, we obtain the global mixed formulation:

$$
\left\{\begin{array}{l}
\text { find }(\Lambda, X) \in \mathcal{M} \times \mathcal{V} \text { such that } \\
\forall Y \in \mathcal{V}, \quad A(X, Y)+B(\Lambda, Y)=L(Y), \\
\forall \Xi \in \mathcal{M}, \quad B(\Xi, X)=0,
\end{array}\right.
$$

where the unknows are $X=\left(u_{\alpha}, u_{3}, \theta_{\alpha}\right)$ and $\Lambda=(\varphi, \psi)$ associated with the virtual fields $Y$ and $\Xi$. Let us remark that, from now on, $u_{\alpha}, u_{3}$ and $\theta_{\alpha}$ are considered as independent variables. Moreover, $L(Y)$ is the natural extension of the linear form $l(v)$ appearing in (6). The bilinear form $A$ reads

$$
\begin{aligned}
A(X, Y) & =\int_{\omega} R^{M \alpha \beta \lambda \mu} \gamma_{\alpha \beta}(X) \gamma_{\lambda \mu}(Y)+\frac{\varepsilon^{2}}{3} \int_{\omega} R^{F \alpha \beta \lambda \mu} \rho_{\alpha \beta}(X) \rho_{\lambda \mu}(Y) \\
& =\int_{\omega} n^{\alpha \beta}(X) \gamma_{\alpha \beta}(Y)+\frac{\varepsilon^{2}}{3} \int_{\omega} m^{\alpha \beta}(X) \rho_{\alpha \beta}(Y)
\end{aligned}
$$

and gives the elastic energy of the shell while $B$, expressed by

$$
\begin{aligned}
B(\Lambda, Y)= & \frac{\varepsilon^{2}}{3}\left[\int_{\omega} g^{\alpha \lambda} \varphi_{, \lambda}\left(\mu_{\alpha}+b_{\alpha}^{\beta} v_{\beta}+v_{3, \alpha}\right)\right. \\
& -\int_{\omega} \frac{\psi}{\sqrt{|g|}}\left(\mu_{2,1}-\mu_{1,2}+\left(b_{2}^{\beta} v_{\beta}\right)_{, 1}-\left(b_{1}^{\beta} v_{\beta}\right)_{, 2}\right]
\end{aligned}
$$

ensures the Kirchhoff-Love constraint (5). As a matter of fact, displacement fields $X$ such that $B(\Xi, X)=0$ for all $\Xi \in \mathcal{M}$, are precisely Kirchhoff-Love displacement fields. Finally, the functional spaces are defined by

$$
\mathcal{V}=V_{t} \times V \times W_{t} \quad, \quad \mathcal{M}=V \times L_{0}^{2}(\omega)
$$


with

$$
\left\{\begin{array}{l}
V_{t}=\left\{v_{t}=v^{\alpha} a_{\alpha} \mid v^{\alpha} \in H^{1}(\omega) ; v^{\alpha}=0 \text { on } \gamma_{0} \cup \gamma_{1}\right\}, \\
V=\left\{v \in H^{1}(\omega) \mid v=0 \text { on } \gamma_{0} \cup \gamma_{1}\right\}, \\
L_{0}^{2}(\omega)=\left\{\psi \in L^{2}(\omega) \mid \int_{\omega} \psi=0\right\}, \\
W_{t}=\left\{\mu=\mu_{\alpha} a^{\alpha} \mid \mu_{\alpha} \in H^{1}(\omega) ; \mu_{\alpha}=0 \text { on } \gamma_{0} ; \mu_{t}=0 \text { on } \gamma_{1}\right\}
\end{array},\right.
$$

where $\mu_{t}$ stands for the tangential component along the edge. Moreover, to make the boundary conditions more precise, $\gamma_{0}$ and $\gamma_{1}$ are two parts of the boundary $\gamma$ of $\omega$, where the shell is assumed to be clamped and simply supported respectively. The rest of the boundary of $\omega$ is free.

- To conclude this section, let us emphasize an advantage of this formulation. We have observed that the classical variational formulation needs the use of the third order derivatives of the mapping $\phi$ describing $\omega$. Here, these derivatives should appear in the bilinear form $B$. But a simple calculus leads to the relation

$$
\left(b_{2}^{\beta} v_{\beta}\right)_{, 1}-\left(b_{1}^{\beta} v_{\beta}\right)_{, 2}=b_{2}^{\beta} v_{\beta, 1}-b_{1}^{\beta} v_{\beta, 2}+\left(\Gamma_{\mu 2}^{\beta} b_{1}^{\mu}-\Gamma_{\mu 1}^{\beta} b_{2}^{\mu}\right) v_{\beta}
$$

and the third order derivatives disappear. This point will be of importance when the geometry of the shell is not exactly known and one has to construct an approximation.

\subsection{Numerical discretization}

- In the case of classical variational formulations, such as (6), the main condition, which ensures existence and uniqueness of a solution, is the ellipticity of 
the bilinear form. So, when a conformal finite element approximation is used, which means that the discrete space is contained in the continuous one, this ellipticity property is kept and the discrete model has also one and only one solution (see [17]). Unfortunately, this condition of conformity of the discrete spaces is not sufficient in the case of mixed formulations. This problem is wellknown in fluid mechanics and is due to the incompressibility condition. A long time ago (for example, see [18]), efficient numerical schemes were suggested to overcome this difficulty. In this case, where the main unknows are velocity and pressure, the idea is to enrich the velocity. We follow this idea, keeping in mind that the field which takes the place of velocity is the transverse section rotation $\theta$.

- Practically, first order degree polynomials are used for each unknown $u_{\alpha}$, $u_{3}, \theta_{\alpha}, \varphi$ and $\psi$. But, in order to stabilize the numerical scheme, the rotation $\theta$ is enriched with internal degrees of freedom. More precisely, if triangular elements are used, which is the case in the following of this paper, a "bubble" function is added for each component of $\theta$. With this scheme, we can prove that the error estimate between the exact solution and the discrete one is of order $\mathcal{O}(h)$, if $h$ stands for the small parameter linked to the mesh size (see [19]). 


\section{Approximation of the middle surface of the shell and refinement strategy \\ 4.1 Approximation of the middle surface}

Now, it is assumed that the middle surface $\omega$ of the shell is approximated by flat triangular elements, the vertices of which are on $\omega$. Moreover, it is assumed that the normal vectors to $\omega$ at these vertices are exactly known (see Figure 2). Then, it is possible to build a local basis, i.e. depending on the element, of the tangent plane at each vertex of the mesh. From an interpolation of these "nodal" bases, one can obtain an approximation of the geometrical tensors (1), (2) and (3), which are needed in the shell model. And it can be proved that the error, due to this approximation, is also of order $\mathcal{O}(h)$ in energy norm, which is the same as the error due to the finite element interpolation (see [20]).

\subsection{Mesh refinement: basic aspects}

Let us begin with the technical aspects of the mesh refinement, recalling that we use meshes only made of triangles. In order to refine these meshes, an algorithm proposed by Rivara is used (see [21]). Its mains ideas are the following ones. In a first time, each triangle which has to be refined, is divided into four sub-triangles, in the following manner (see Figure 3): the middle point of the longest side is connected with its opposite vertex and with the middle points of the two other sides.

Once this first refinement is achieved, a second one is done to ensure the conformity of the new mesh. So, two cases may occur. If a new node is added 
on the longest side of a triangle, it is connected with it opposite vertex. If not, it is connected with the middle point of the triangle longest side (see Figure 4). This process is repeated until total conformity is obtained, which practically occurs after a few iterations. Finally, let us emphasize a major advantage of this technique: during the successive refinements, the final mesh has angles which remain bounded away from zero (see [21]).

\subsection{Mesh refinement: case of shell structures}

- As far as refinement is concerned, we have to deal now with the fact that the mesh to refine is not plane. Thus, the middle point of a triangle edge is generally not on the surface. So, we shall use a methodology developed by one of the authors and a coworker (see [8]). For autonomy of this paper, let us describe this strategy hereafter.

- Let $A$ and $B$ be two vertices of the mesh, which are then on the middle surface $\omega$, and $N(A)$ and $N(B)$ the corresponding unit normal vectors. Points $A, B$ and the vector $\gamma \equiv N(A)+N(B)$ define a plane $\Pi$ which cuts $\omega$

along a curve, say $\overparen{A B}$, that we are aiming at defining an interpolation. First, setting $U=\frac{A B}{\|A B\|}$, a direct orthonormal basis $(U, V)$ of $\Pi$ is defined. Then, two vectors $T(A)$ and $T(B)$ are introduced, which belong to plane $\Pi$, and are respectively orthogonal to $N(A)$ and $N(B)$, in such a way that the two vectors $T$ and $N$ define a direct orthonormal basis (see Figure 5 ). So, they are tangent to the curve we are trying to construct an interpolation. Now, let $k$ be the euclidian norm of vector $A B$. We build the third order polynomial function, say $P_{3}$, from $[0, k]$ into $\mathbb{R}$, such that its curve in $\Pi$ contains $A$ and $B$, and 
has $T(A)$ and $T(B)$ as tangent vectors at these points. In other words,

$$
P_{3}(0)=P_{3}(k)=0 \quad, \quad P_{3}^{\prime}(0)=\frac{T_{v}(A)}{T_{u}(A)} \equiv \alpha \quad, \quad P_{3}^{\prime}(k)=\frac{T_{v}(B)}{T_{u}(B)} \equiv \beta,
$$

where $T_{u}$ and $T_{v}$ stand for the components of vector $T$ in the basis $(U, V)$. Then, it is easy to calculate $P_{3}$ :

$$
P_{3}(x)=\alpha x-\frac{2 \alpha+\beta}{k} x^{2}+\frac{\alpha+\beta}{k^{2}} x^{3} .
$$

Finally, the new node $C$ is introduced such that:

$$
A C=\frac{k}{2} U+P_{3}\left(\frac{k}{2}\right) V=\frac{k}{2} U+\frac{k}{8}(\alpha-\beta) V
$$

Remark. In general, the two vertices $A$ and $B$, and the two normal vectors $N(A)$ and $N(B)$ are not in the same plane. So, on Figure $5, \tilde{N}(A)$ and $\tilde{N}(B)$ stand for the projection of the exact normal vectors on the plane $\Pi$ and are given only for the understanding of the Figure. They are useless for building the function $P_{3}$.

- Let us now assume that the exact curve $\overparen{A B}$ can be described by a mapping $f$, which is assumed to be $\mathcal{C}^{4}([0, k])$. Then, the previous interpolation procedure is reduced to the well-known Hermite interpolation, for which the error is classical. For all $x \in[0, k]$, there exists $\left.\xi_{x} \in\right] 0, k[$ such that:

$$
f(x)-P_{3}(x)=\frac{x^{2}(x-k)^{2}}{24} f^{(4)}\left(\xi_{x}\right)
$$

Therefore, if $M$ is a general node of $\widehat{A B}$ and $M_{h}$ the node obtained by the above interpolation, the two nodes being of same abscissa in $(A ; U, V)$, one has

$$
\left\|M M_{h}\right\|_{L^{\infty}([0, k])} \leq \eta_{k} k^{4}
$$


where $\eta_{k}$ is a constant independent on $k$ but depending on the maximum value of $\left|f^{(4)}\right|$ on $[0, k]\left(f^{(4)}\right.$ is the fourth order derivative of $\left.f\right)$. So, when we choose for $M$ the node of abscissa $k / 2$, we have $\|M C\| \leq \eta_{k} k^{4}$. Finally, introducing the mesh parameter $h$, which is the maximum length of the edges in the mesh, and assuming that the mapping is sufficiently smooth, we can conclude that there exists a strictly positive constant $\eta$, independent on $h$ and such that, on the whole mesh:

$$
\|M C\| \leq \eta h^{4},
$$

where $C$ stands for the added nodes corresponding to the "exact" nodes $M$.

- Nevertheless, the geometrical approximation needs also the use of the normal vector. So, we have to define a unit normal vector to $\omega$ at node $C$. For this, the classical linear interpolation is used and we normalize the vector that has been obtained:

$$
N(C)=\frac{N(A)+N(B)}{\|N(A)+N(B)\|} .
$$

In order to obtain an error estimate between $N(C)$ and the exact normal vector $N(M)$ ( $M$ is again the node of abscissa $k / 2$, previously introduced), let us recall a classical interpolation estimate, applied to our case:

$$
\|N-\pi N\|_{L^{\infty}(\omega)} \leq c h^{2}|N|_{W^{2, \infty}(\omega)}
$$

if $\pi$ stands for the linear interpolation operator, under classical regularity assumptions on the mesh and if the components of $N$ belongs to $W^{2, \infty}(\omega)$ (see [17]). $N$ represents the unit normal vector. It is homogeneous to the first order derivatives of the mapping $\phi$. Therefore, it is smooth enough because the mapping which defines the surface $\omega$ has been assumed to be $\mathcal{C}^{3}(\widehat{\omega})$. Let us 
also note that the normal vector is defined everywhere on the middle surface $\omega$, because the two tangent vectors have been assumed to be linearly independent at each point of the surface $\omega$. From the definition of $M$ ("middle" point of $\widehat{A B})$, one has

$$
\pi N(M)=\frac{N(A)+N(B)}{2} .
$$

Therefore

$$
\begin{aligned}
\|\pi N(M)\| & =\|N(M)-N(M)+\pi N(M)\| \\
& \geq\|N(M)\|-\|N(M)-\pi N(M)\| \\
& \geq\|N(M)\|-\|N-\pi N\|_{L^{\infty}(\omega)} \\
& \geq 1-c h^{2}|N|_{W^{2, \infty}(\omega)},
\end{aligned}
$$

or else

$$
1-\|\pi N(M)\| \leq c h^{2}|N|_{W^{2, \infty}(\omega)}
$$

Moreover, one has

$$
\begin{aligned}
\|N(M)-N(C)\| & \leq\|N(M)-\pi N(M)\|+\|\pi N(M)-N(C)\| \\
& \leq\|N-\pi N\|_{L^{\infty}(\omega)}+\|\pi N(M)-N(C)\| .
\end{aligned}
$$

Let us remark now that

$$
\begin{aligned}
\|\pi N(M)-N(C)\| & =\left\|\pi N(M)-\frac{\pi N(M)}{\|\pi N(M)\|}\right\| \\
& =|\|\pi N(M)\|-1|=1-\|\pi N(M)\|,
\end{aligned}
$$

as

$$
\|\pi N(M)\|=\frac{\|N(A)+N(B)\|}{2} \leq 1
$$

because the normal vectors are unitary. Finally, using (14), we obtain

$$
\|N(M)-N(C)\| \leq 2 c h^{2}|N|_{W^{2, \infty}(\omega)}
$$


- The previous method was applied on various surfaces, and the numerical results coroborate the above estimates (see [8]).

Remark. Let us observe that this procedure only requires data which are connected to the element edge (coordinates of the two vertices and the two associated normal vectors). It doesn't depend on the fact that the element is a triangle or a quadrangle. So, it could be applied exactly in the same way for refining meshes made of quadrangles.

\section{Mesh refinement criteria}

We will now explain how we choose to refine the mesh. To make it short, the method is based on the smoothing of the elastic energy of the shell. So, a first step is to build new fields of stresses which are supposed to give a better approximation of the true ones than the finite element ones. Then, a first part of an error indicator can be obtained thanks to the difference between smoothed and finite element fields. Nevertheless, as we work on shell models, we have chosen to introduce in our indicator the transverse shear stress as a quantity of interest, in a way which is described in the following. On the other hand, though it could certainly be interesting in the framework of shells, in the present paper, we have not introduced an indicator linked with the quality of the geometry approximation, as it is suggested in [7]. Nevertheless, as it will appear further, in most of our test cases, we have studied the effect of this approximation. 


\subsection{Stress smoothing}

- First of all, let us notice that the finite element membrane stress $n^{h}$ and bending moment $m^{h}$ are constant on each triangle, as first-order polynomial functions are used for the displacements. Moreover, as they are given in a local basis which depends on the element, they have to be expressed in the same global basis $\left(e_{1}, e_{2}, e_{3}\right)$ before being smoothed. Then, let us denote by $n_{k l}^{h}(K)$ and $m_{k l}^{h}(K)$ the values which are taken on triangle $K$ by any component of $n^{h}$ and $m^{h}$ respectively. Then, at any vertex $S$ of the mesh, we define for any component $k$ and $l$ :

$$
n_{k l}^{h}(S)=\frac{\sum_{\mathcal{T}_{S}}|K| n_{k l}^{h}(K)}{\sum_{\mathcal{T}_{S}}|K|} \quad, \quad m_{k l}^{h}(S)=\frac{\sum_{\mathcal{T}_{S}}|K| m_{k l}^{h}(K)}{\sum_{\mathcal{T}_{S}}|K|}
$$

where $\mathcal{T}_{S}$ denotes the patch of $S$, which means the set of elements $K$ which con-

tain the vertex $S$. Then, the smoothed membrane stress $\widetilde{n}_{K}^{h}$ and the smoothed bending moment $\widetilde{m}_{K}^{h}$ are defined as the tensors any component of which is the first-order polynomial functions given on each triangle $K$ by

$$
\left\{\begin{array}{l}
\widetilde{n}_{k l}^{K}=\sum_{i=1}^{3} n_{k l}^{h}\left(S_{i}\right) \lambda_{i}^{K} \\
\widetilde{m}_{k l}^{K}=\sum_{i=1}^{3} m_{k l}^{h}\left(S_{i}\right) \lambda_{i}^{K}
\end{array}\right.
$$

where $\lambda_{i}^{K}$ stands for the $i^{\text {th }}$ first-order nodal function of $K$.

Remark. The values given by (15) are solution of

$$
\min _{f \in \mathbb{R}} \int_{\mathcal{T}_{S}}\left(f-f_{K}(X)\right)^{2} d X
$$

where $f_{K}(X)$ is a generic function, which is constant on each element, as in the case of $n^{h}$ and $m^{h}$. Thus, our approach presents several analogies with 
the one developed by Zhu and Zienkiewicz in [9] and [10]. Let us note yet that we minimize among constants while these authors use first-order degree polynomials.

\subsection{Error indication}

- Let us first recall some basic aspects on the elastic energy $J$ of a general shell model. Let $\zeta$ be the linearised strain tensor and $\sigma$ the stress tensor. The three-dimensional elastic energy is given by

$$
J=\frac{1}{2} \int_{\Omega} \sigma: \zeta
$$

Now, the tensors $\sigma$ and $\zeta$ can be splitted into tangential and normal components. We have:

$$
\zeta=\zeta_{t}+\zeta_{s} \otimes N+N \otimes \zeta_{s}+\zeta_{n} N \otimes N
$$

and a similar equation for $\sigma$. Then, one derives the following decomposition of the elastic energy:

$$
J=J_{t}+J_{s}+J_{n}
$$

with

$$
J_{t}=\frac{1}{2} \int_{\Omega} \sigma_{t}: \zeta_{t} \quad, \quad J_{s}=\int_{\Omega} \sigma_{s} \cdot \zeta_{s} \quad, \quad J_{n}=\frac{1}{2} \int_{\Omega} \sigma_{n} \zeta_{n} .
$$

The two first quantities are respectively the inplane and the transverse shear energies. 
- In order to recover error indicators homogeneous to an energy, the next step we consider is to express some terms of the decomposition (18) using the stresses we are dealing with. Let us first notice that the kinematical hypothesis of the Koiter's shell model leads to

$$
\zeta_{t}=\gamma+x_{3} \rho
$$

$x_{3}$ being the coordinate along the normal direction to the middle surface. Then, we get for the inplane strain energy, which is the global elastic energy for our shell,

$$
J_{t}=\frac{1}{2} \int_{\omega} n:\left(R^{M}\right)^{-1}: n+\frac{\varepsilon^{2}}{6} \int_{\omega} m:\left(R^{F}\right)^{-1}: m
$$

- Such an expression is appropriate for the smoothing strategy presented in the previous section, and leads naturally to a global error control. But here, we have also chosen to enrich our error indicator with the transverse shear stress. By the way, because of Kirchhoff-Love assumptions, there is no transverse shear energy for the Koiter's or/and Budiansky-Sanders's models. This is due to the fact that the transverse shear strain is zero. Let us observe that Kirchhoff-Love assumptions can be justified thanks to asymptotic methods. It means that the most the shell is thin, the most the transverse shear energy is close to zero. Hence, introducing an energy for the transverse shear in the framework of Kirchhoff-Love's model could appear as not justified in a first time. Nevertheless, first, the "real" thickness of the shells on which engineers work, doesn't go to zero. The thickness can be very small but is not null. And second, in our model, we can use the expression of the Lagrange multiplier to give an indicator on the transverse shear energy. This quantity is of importance when the transverse shear stress takes locally a big value: it is a way to mean 
that the shell model becomes questionable and a manner to overcome this, is to refine the mesh locally there. So, from a three-dimensional constitutive law, we define a resultant transverse energy by setting:

$$
I(K)=\frac{1+\nu}{2 E \varepsilon}\|\operatorname{grad} \varphi+\operatorname{rot} \psi\|_{L^{2}(K)}^{2} \equiv \frac{1+\nu}{2 E \varepsilon}\left\|q^{h}\right\|_{L^{2}(K)}^{2}
$$

where $\varphi$ and $\psi$ are the two potential functions used in the definition of the transverse shear stress (see $(7))$.

- The previous considerations lead us to define error indicators for each type of stresses by

$$
\left\{\begin{array}{l}
\eta_{n}(K)=\sqrt{\frac{1-\nu^{2}}{2 E \varepsilon}}\left\|\widetilde{n}^{h}-n^{h}\right\|_{L^{2}(K)} \\
\eta_{m}(K)=\sqrt{\frac{\varepsilon\left(1-\nu^{2}\right)}{6 E}}\left\|\widetilde{m}^{h}-m^{h}\right\|_{L^{2}(K)}, \\
\eta_{q}(K)=\sqrt{I(K)}
\end{array}\right.
$$

In order to define a relative error indicator, we introduce the positive quantity

$$
\begin{aligned}
J_{0}=\sum_{K \in \mathcal{T}_{0}} & \left\{\frac{1-\nu^{2}}{2 E \varepsilon}\left\|\widetilde{n}^{h}\right\|_{L^{2}(K)}^{2}\right. \\
& \left.+\frac{\varepsilon\left(1-\nu^{2}\right)}{6 E}\left\|\widetilde{m}^{h}\right\|_{L^{2}(K)}^{2}+\frac{1+\nu}{2 E \varepsilon}\left\|q^{h}\right\|_{L^{2}(K)}^{2}\right\},
\end{aligned}
$$

where $\mathcal{T}_{0}$ is the mesh used for the initial computation. The local error indicator is then

$$
\eta(K)=\sqrt{\frac{\eta_{n}^{2}(K)+\eta_{m}^{2}(K)+\eta_{q}^{2}(K)}{J_{0}}}
$$


- Finally, the refinement of a triangle $K$ is effective when one has, for a given positive or null $\alpha$,

$$
\eta(K)>\eta_{\text {mean }}+\alpha s
$$

where $\eta_{\text {mean }}$ and $s$ stand respectively for the arithmetic mean and standard deviation of error indicators. In general, the larger is $\alpha$, the more concentrated will be mesh refinement. In fact, the values of this parameter influence the decrease of the global estimation during the refinement process, as shown later.

\section{Numerical experiments}

We present here some numerical results testing the approach previously described. Among them are classical shell problems, for which reference solutions are described in the literature (see e.g. [22]). They allow us to check the computation accuracy when the number of elements increases while refining the mesh. In particular, we will examine the following points, which we found interesting in our approach.

- What is the effect of the geometry approximation on the computation accuracy after a few refinement iterations?

Thus, when it is possible, it will be worthwhile to consider the case of the exact interpolation in order to compare the results with the approximated interpolation ones.

- Are the error indicators of the different stresses such that each of them can play a role in the refinement process? 
If it is, it will justify the weights chosen in (22).

- Is the error indicators behaviour close to the one of the "true" errors?

This can be investigated when an analytical solution of the problem is available, as in the first case considered underneath. It should be noticed that the effectivity index is classically used to answer to this question (see e.g. [23]). Let us remember that the main goal of the indicator suggested here is to detect where to refine in order to obtain a good accuracy for the transverse shear stress. It is not designed to be close to the "true" error.

\subsection{Cylinder under uniform pressure}

- Let us begin with the case of a cylindrical shell, which is clamped on one side, the opposite one being free. It is submitted to an internal uniform pressure load $P$. Thus, the shell and the load are both axisymmetric. So, the solution depends only on the abscissa along the shell axis, say $\xi^{2}$, in the case of the mapping

$$
\phi\left(\xi^{1}, \xi^{2}\right)=\left(\begin{array}{c}
\xi^{2} \\
R \cos \xi^{1} \\
R \sin \xi^{1}
\end{array}\right)
$$

where $R$ is the radius of the cylinder. The main interest of this example is that the exact solution of the Koiter's model can be easily obtained (see [24]). 
- In the local basis given by (25), the membrane stress tensor $n$ is such that

$$
n_{1}^{1}=\frac{2 E \varepsilon}{R} u_{3},
$$

its other components being zero, and the bending moments are

$$
\left\{\begin{array}{l}
m_{1}^{1}=-\frac{2 E \varepsilon}{1-\nu^{2}}\left(\frac{1}{R^{2}} u_{3}+\nu u_{3,22}\right), \\
m_{2}^{2}=-\frac{2 E \varepsilon}{1-\nu^{2}}\left(\frac{\nu}{R^{2}} u_{3}+u_{3,22}\right),
\end{array}\right.
$$

while $m_{1}^{2}=m_{2}^{1}=0$. Concerning the transverse shear stress, one has

$$
q^{1}=0 \quad, \quad q^{2}=m_{, 2}^{22} .
$$

Finally, the knowledge of the deflection $u_{3}$ allows us to compute all these stresses. This can be done by solving the following differential equation:

$$
\left\{\begin{array}{l}
\frac{2 E \varepsilon^{3}}{3\left(1-\nu^{2}\right)}\left\{u_{3,2222}+\frac{2 \nu}{R^{2}} u_{3,22}+\left(\frac{1}{R^{2}}+\frac{3\left(1-\nu^{2}\right)}{\varepsilon^{2}}\right) \frac{u^{3}}{R^{2}}\right\}=P \\
u_{3}(L)=0, \quad u_{3,2}(L)=0, \quad m_{2}^{2}(0)=0, \quad m_{2,2}^{2}(0)=0
\end{array},\right.
$$

where $L$ is the cylinder length. The settings for the computation are given in Table 1.

- One observes in Figures 6 to 9 the very good accuracy of the numerical solution versus the analytical one, after four refinements, even for the bending moment and the transverse shear stress, especially near the clamped edge. This case clearly illustrates the fact that the transverse shear stress doesn't vanish in the Kirchhoff-Love's model: it is close to zero on most of the shell but far from it near the clamped edge (see Figure 9). 
- Finally, one can derive an approximated "true" error for this test-problem. To achieve this, the difference between the analytical values of the stresses at the vertices of the mesh and the finite element stresses is evaluated in a similar way than in (21)-(22). More precisely, we set for each triangle $K$ :

$$
\left\{\begin{array}{l}
\widetilde{n}_{K}^{a}=\sum_{i=1}^{3} n_{S_{i}}^{a} \lambda_{i}^{K} \\
\widetilde{m}_{K}^{a}=\sum_{i=1}^{3} m_{S_{i}}^{a} \lambda_{i}^{K} \\
\widetilde{q}_{K}^{a}=\sum_{i=1}^{3} q_{S_{i}}^{a} \lambda_{i}^{K}
\end{array}\right.
$$

where the superscript $a$ denotes the analytical solution. Then, the approximated "true" error is defined as

$$
e(K)=\sqrt{\frac{e_{n}^{2}(K)+e_{m}^{2}(K)+e_{q}^{2}(K)}{J_{0}^{a}}},
$$

where

$$
\left\{\begin{array}{l}
e_{n}(K)=\sqrt{\frac{1-\nu^{2}}{2 E \varepsilon}}\left\|\widetilde{n}^{a}-n^{h}\right\|_{L^{2}(K)} \\
e_{m}(K)=\sqrt{\frac{\varepsilon\left(1-\nu^{2}\right)}{6 E}}\left\|\widetilde{m}^{a}-m^{h}\right\|_{L^{2}(K)} \\
e_{q}(K)=\sqrt{\frac{1+\nu}{2 E \varepsilon}}\left\|\widetilde{q}^{a}-q^{h}\right\|_{L^{2}(K)}
\end{array}\right.
$$

and

$$
\begin{aligned}
J_{0}^{a}=\sum_{K \in \mathcal{T}_{0}} & \left\{\frac{1-\nu^{2}}{2 E \varepsilon}\left\|\widetilde{n}^{a}\right\|_{L^{2}(K)}^{2}\right. \\
& \left.+\frac{\varepsilon\left(1-\nu^{2}\right)}{6 E}\left\|\widetilde{m}^{a}\right\|_{L^{2}(K)}^{2}+\frac{1+\nu}{2 E \varepsilon}\left\|\widetilde{q}^{a}\right\|_{L^{2}(K)}^{2}\right\}
\end{aligned}
$$

Figure 11 allows one to compare the error indicators with the estimated "true" errors, as the mesh is refined. The decrease rate of the quadratic mean values 
of the errors is more important than the one of the indicators. This behaviour is due to the fact that the tranverse shear stress is compared to zero in our indicator. However, it is locally very large near the clamped edge (see Figure 9). Then, the mixed model introduced in Section 3 combined with our mesh refinement criteria allows one to refine the mesh where the transverse shear stress is important and to obtain a good accuracy on it. Moreover, Figure 11 illustrates also the efficiency of the mesh refinement compared to a uniform mesh refinement.

- The comparison of Figures 10 to 14 shows how the selectivity parameter $\alpha$ introduced in (24) influences the decrease rate of the error indicators and the "true" errors. For large values of $\alpha$, the decrease rate can be irregular.

- Finally, one can describe more precisely the effects of each part of the error indicator. Thus, $\eta_{n} / \sqrt{J_{0}}, \eta_{m} / \sqrt{J_{0}}, \eta_{q} / \sqrt{J_{0}}$ and the global indicator $\eta$ along the axis direction, for the initial computation and after 1,2 and 3 refinement iterations, are shown in Figures 15, 16, 17 and 18. In all the cases, most of the error concentrates near the clamped edge. Nevertheless, the effect of each indicator doesn't follow the same way. The membrane stress indicator rapidly decreases near the clamped edge and remains dominant far from it. Near the clamped edge, the transverse shear stress indicator becomes more and more important, relatively to the two other ones, and finally select the refinement area. The effect of the bending moment indicator is rather weak. It also decreases rapidly near the clamped edge and increases near the free edge. Let us observe that dropping the transverse shear stress indicator away from the global indicator would completely change the refinement strategy as the global error would concentrate far from the clamped edge. 


\subsection{Pinched cylinder}

- Here, a cylindrical shell is simply supported at its extremities by two diaphragms and loaded by two opposite pointwise forces $F$ and $-F$ in the middle. Because of the problem symmetry, only one eighth of the cylinder is generally considered where the force $F / 4$ is applied (see Figure 19).

- An analytical solution, which is used as a reference one for this problem, is obtained using a double Fourier series and the relations due to Flügge (see [25]). But it doesn't correspond exactly to the Koiter's model, for which the change of curvature tensor is slightly different. So, this so-called reference value

for the normalized deflection at the pinched point is $\frac{2 E \varepsilon u_{3}}{\|F\|}=-164.24$. When starting from a uniform mesh of 400 elements, we get -164.21 at the $7^{\text {th }}$ computation (see Figure 20). Let us note that this value doesn't vary significantly when using finer meshes. Therefore, one can say that the convergence has been obtained for our model.

Analogously, concerning the stresses, a reference normalized value of the component $n_{11}$ of the membrane stress in the absolute basis at the pinched point is $\frac{R n_{11}}{\|F\|}=-15.72$, while we get -17.3 . Keeping in mind that these results arise from different shell models, the relatively small differences observed lead us to consider our results as reliable, for both the displacements and the stresses.

- The approximated interpolation of vertices and normal vectors, described in Section 4.3, generates a neglectible error for the deflection, as shown in Figure 21. It is worth to note that the conclusion is the same for the stresses (see Figure 22). 
- Finally, the error indicators $\eta_{n} / \sqrt{J_{0}}, \eta_{m} / \sqrt{J_{0}}, \eta_{q} / \sqrt{J_{0}}$ along $\overparen{A B}$ and the global indicator $\eta$ are shown in Figure 23. One observes that the membrane stress indicator is dominant away from the pinched point, while near this point the bending moment and the transverse shear stress indicators grow and finally contribute to select the refinement area. The same features remain valid after more refinement iterations (see Figure 24). For these computations, our goal is reached because $\eta_{n}, \eta_{m}$ and $\eta_{q}$ are weighted in such a manner that none of them masks systematically the others during the refinement process. A last point that should be noticed is the efficiency of the mesh refinement versus a uniform mesh refinement, as shown in Figure 25.

\subsection{Pinched twisted ribbon}

- Let us now consider another instructive classical example: the twisted cantilevered ribbon (see Figure 26). One extremity is clamped and the opposite one is submitted to a concentrated unit shear-load in the thickness direction.

- The computed displacement for the pinched point $A$ in the basis $\left(e_{1}, e_{2}, e_{3}\right)$ is $(0 ., 0.00175,-0.00171)$ at the $5^{\text {th }}$ computation as the reference solution given by $[22]$ is $(0 ., 0.00175,-0.00179)$, this solution being derived from the beam theory.

- The error indicators $\eta, \eta_{n} / \sqrt{J_{0}}, \eta_{m} / \sqrt{J_{0}}$ and $\eta_{q} / \sqrt{J_{0}}$ along $\overparen{D E}$ are shown in Figure 28. One observes that the transverse shear and the membrane stress indicators lead to the same order of magnitude. Although the bending moment drops back in this case, the weights of (22) are therefore satisfying.

- Finally, one observes in Figure 27 the good accuracy along $\overparen{D E}$ of the ap- 
proximated interpolation method proposed in [8], as Figure 29 reveals, at the pinched point, the approximated interpolation gives slightly less accurate results than the exact one. To overcome this problem, it could be advantageous to incorporate some shell geometrical informations to the error indicator (see [7] for an example of such an approach).

\subsection{Clamped hyperbolic paraboloid}

- The middle surface is given by the mapping

$$
\phi\left(\xi^{1}, \xi^{2}\right)=\left(\begin{array}{c}
\xi^{1}-b \\
\xi^{2}-b \\
\frac{c}{2 b^{2}}\left(\left(\xi^{2}\right)^{2}-\left(\xi^{1}\right)^{2}\right)
\end{array}\right)
$$

where $b$ and $c$ are two positive numbers and $\widehat{\omega}=] 0,2 b[\times] 0,2 b[$ (see Figure 30, where the frame $O x^{\prime} y^{\prime}$ is obtained from $O x y$ by the rotation of $\pi / 4$ radians in this plane). The geometrical and mechanical settings are those of the case described in e.g. [26].

- First, let us notice that the displacement of the center point $O$ is close to the reference solution given in [26]. For the clamped parabolic hyperboloid, the refinement concentrates near the boundaries. The clamped boundary condition, which creates a horizontal tangency along the edge, appears clearly on the deformed configuration after few refinements, as shown in Figure 31. Again, exact and approximated interpolations give very close results. 
- Figures 32 and 33 illustrate that the refinement process is strongly dependant on the transverse shear stress indicator $\eta_{q}$ for the clamped hyperbolic paraboloid. This underlines again the importance of $\eta_{q}$ in the expression (23) of the error indicator.

\subsection{A case of junction of shells: tank under uniform pressure}

- As a more complex structure than the previous ones, we consider now a shell which contains junctions between a cylinder and two hemispheres. The mechanical load is an internal uniform pressure. The data are given in Tables 2 and 3. Due to the problem symmetry, only one eighth of the structure is considered (see Figure 34).

- An analytical solution is available in [24] for the tank under uniform pressure. However, the hemispheres are considered as two membranes and the shell model of the cylinder is Novozhilov's one, which is different from Koiter's model. Consequently, this analytical result is too different from ours to make a relevant quantitative comparison. However, it indicates that the deflection variation has to be located at the junction. This can be observed in Figure 35. Furthermore, one can observe in Figure 36, as expected, the location of the refinement in the vicinity of the junction. Correspondingly, the error indicators concentrate on the junction during the refinement process (see Figure 37, the results remain the same after several iterations). Finally, one observes in Figure 38 the good results of the approximated interpolation and the interest of a refinement strategy versus a uniform mesh refinement. 


\subsection{A case of cracked shell}

- The last test-problem considered in this paper is a cracked cylindrical shell. One quarter of a cylinder is clamped on all its boundary and submitted to a uniform pressure. The settings are those of the Table 1. Let us note that the crack was chosen so that its direction is not along the principal directions of curvature of the middle surface (see Figure 39).

- It can be observed in Figure 40 that the refinement propagates partially in an orthogonal direction of the crack.

- All error indicators decrease near the crack tip during the refinement process (see Figure 41) and it is interesting to notice that, in this case, the membrane stress indicator is highly dominant. In the studied case, the opening mode is Mode I, for which the stresses are the most important in a direction which is perpendicular to the crack: it explains the refinement propagation. Finally, it can be observed that, as expected, the stresses increase near the crack tip, which is in fact theoretically singular (see Figure 42). Here the stresses are evaluated in $L^{2}$ norm on the patch of the crack tip.

\section{Conclusion}

A fundamental problem arising in shell structure approximation, is the geometry. Even if it is known from an analytical expression, it implies a lot of numerical difficulties in the approximation. A first step is to use the definition of a set of vertices displayed on the middle surface of the shell and to assume that the exact normal are known at these points. A consistent numer- 
ical scheme can be developed from these reduced informations on the shell geometry $[16,20]$. But, as in the non-linear analysis, when one tries to add new points during a mesh refinement procedure, it becomes necessary to introduce a local approximation of the surface along the edges of each element, in order to define with precision the new points and the new normals at these points. The important feature is that the true informations on the real middle surface of the shell are not necessarily known. Therefore, it has been useful to adapt the strategy introduced in [8] to adaptive mesh refinements. The results obtained in this paper show that the method works with accuracy. As illustrated on various different shells, the effect of geometry interpolation on the mesh refinement procedure is neglectible. Moreover, the introduction, in a more classical global indicator, of the transverse shear stress indicator, as a quantity of interest in the case of shell structures, has been investigated. Its influence can be determinant in the refinement strategy. A further step would be to study more deeply this phenomenon and to introduce other mesh refinement criteria.

\section{References}

[1] A. Pica, R.D. Wood, A.O. Adekunle, and J. Bonet. Smoothing stress resultants in adaptive finite element shell analysis. Computers \& Structures, 54(5):835$849,1995$.

[2] T.J. Bond, L.Y. Li, P. Bettess, J.W. Bull, and I. Applegarth. Adaptive mesh refinement for shells with modified Ahmad elements. Computers $\&$ Structures, 61(6):1135-1141, 1996.

[3] C.S. Han and P. Wriggers. An h-adaptive method for elasto-plastic shell 
problems. Comput. Methods Appl. Mech. Engrg., 189:651-671, 2000.

[4] R. Lackner and H.A. Mang. Mesh generation and mesh refinement procedures for the analysis of concrete shells. Advances in Engineering Software, 33:389402, 2002.

[5] M. Cho and S. Jun. r-adaptive mesh generation for shell finite element analysis. Journal of Computational Physics, 199:291-316, 2004.

[6] T.J.R. Hughes, J.A. Cottrell, and Y. Bazilevs. Isogeometric analysis: CAD, finite elements, NURBS, exact geometry and mesh refinement. Comput. Methods Appl. Mech. Engrg., 194:4135-4195, 2005.

[7] M. Baumann and K. Schweizerhof. Adaptive mesh generation on arbitrarily curved shell structures. Computers \& Structures, 64(1-4):209-220, 1997.

[8] I.A. Arregui and M. Salaün. Interpolation d'une surface déterminée par des nœuds et des normales. C. R. Acad. Sci. Paris, Série I, 318:265-268, 1994.

[9] J.Z. Zhu and O.C. Zienkiewicz. The superconvergent patch recovery and $a$ posteriori error estimates. Part 1: The recovery technique. Int. J. Numer. Meth. Engng, 33:1331-1364, 1992.

[10] J.Z. Zhu and O.C. Zienkiewicz. The superconvergent patch recovery and $a$ posteriori error estimates. Part 2: Error estimates and adaptivity. Int. J. Numer. Meth. Engng, 33:1365-1382, 1992.

[11] F. Cirak and E. Ramm. A posteriori error estimation and adaptivity for linear elasticity using the reciprocal theorem. Comput. Methods Appl. Mech. Engrg., 156:351-362, 1998.

[12] S. Prudhomme, J.T. Oden, T. Westermann, J. Bass, and M.E. Botkin. Practical methods for a posteriori error estimation in engineering applications. Int. J. Numer. Meth. Engng, 56:1193-1224, 2003. 
[13] M. do Carmo. Differential Geometry of Curves and Surfaces. Prentice-Hall, 1976.

[14] W.T. Koiter. On the foundations of the linear theory of thin elastic shells. Proc. Kon. Ned. Akad. Wetensch, B73:169-195, 1970.

[15] B. Budiansky and J.L. Sanders. On the best first-order linear shell theory. Progr. in Appl. Mech., W. Prager Anniversary Volume:129-140, 1967.

[16] Ph. Destuynder and M. Salaün. A mixed finite element for shell model with free edge boundary conditions - Part 1: The mixed variational formulation. Comput. Methods Appl. Mech. Engrg., 120:195-217, 1995.

[17] P.G. Ciarlet. The Finite Element Methods for Elliptic Problems. North-Holland, 1987.

[18] V. Girault and P.-A. Raviart. Finite Element Methods for Navier-Stokes Equations, volume 5 of Springer Series in Computational Mathematics. Springer, Berlin, 1986. Theory and algorithms.

[19] Ph. Destuynder and M. Salaün. A mixed finite element for shell model with free edge boundary conditions - Part 2: The numerical scheme. Comput. Methods Appl. Mech. Engrg., 120:219-242, 1995.

[20] Ph. Destuynder and M. Salaün. Approximation of shell geometry for nonlinear analysis. Comput. Methods Appl. Mech. Engrg., 152:393-430, 1998.

[21] M.C. Rivara. Algorithms for refining triangular grids suitable for adaptive and multigrid techniques. Int. J. Numer. Meth. Engng, 20:745-756, 1984.

[22] J.L. Batoz and G. Dhatt. Modélisation des structures par éléments finis Volume 3 : Coques. Hermès, Paris, 1992.

[23] M. Ainsworth and J.T. Oden. A Posteriori Error Estimation in Finite Element Analysis. Wiley, New York, 2000. 
[24] Ph. Destuynder. Modélisation des coques minces élastiques. Masson, Paris, 1990.

[25] W. Flügge. Stresses in Shells - Second Edition. Springer, 1973.

[26] M. Bernadou. Méthodes d'éléments finis pour les problèmes de coques minces. Masson, Paris, 1994. 


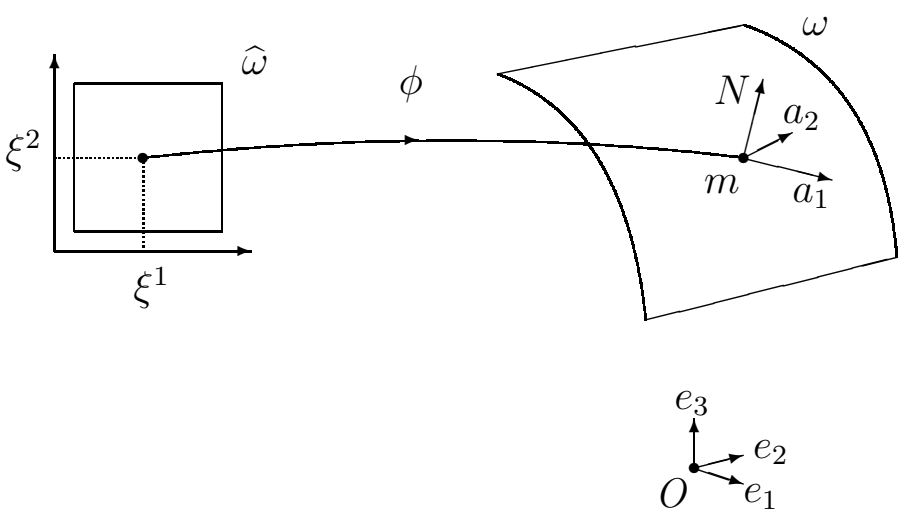

Fig. 1. Definition of surface $\omega$. 


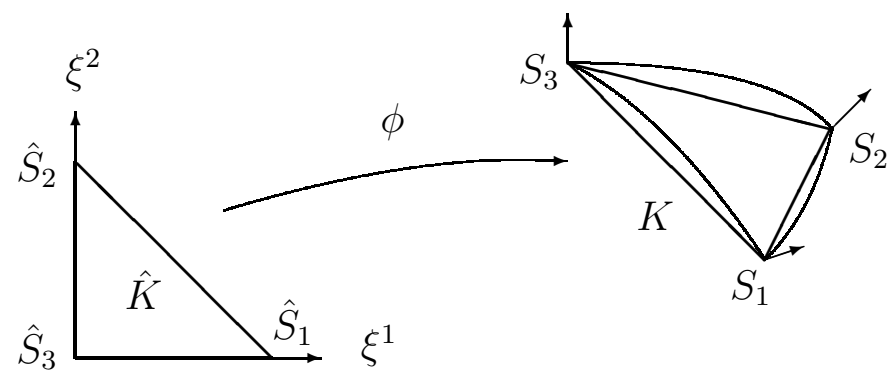

Fig. 2. Approximation of the middle surface geometry. 


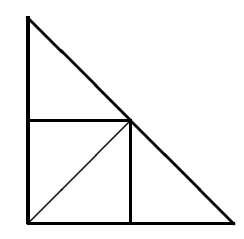

Fig. 3. First refinement of a triangle.

12

13

14

15

16

17

18

19

20

21

22

23

24

25

26

27

28

29

30

31

32

33

34

35

36

37

38

39

40

41

42

43

44

45

46

47

48

49

50

51

52

53

54

55

56

57

58

59

60

61

62

63

64

65 


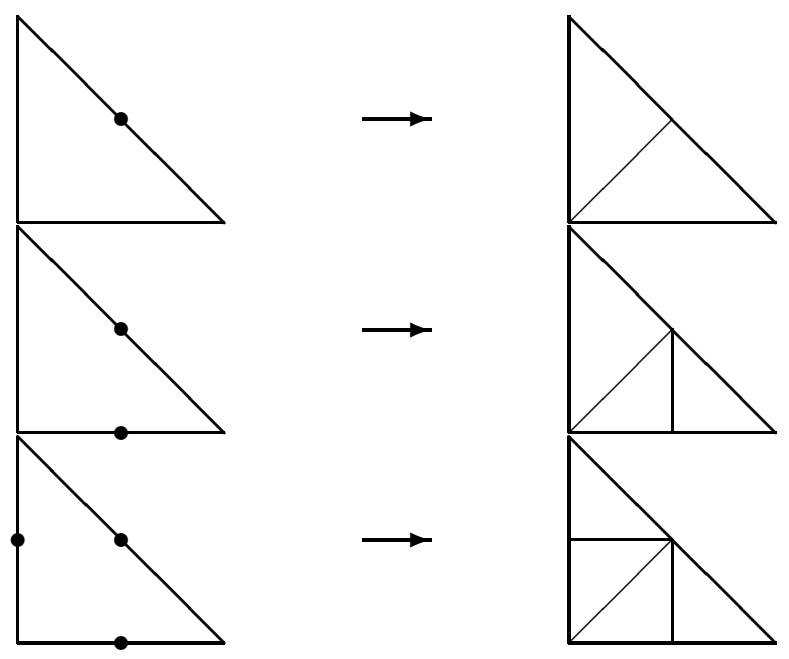

Fig. 4. Next refinements of a triangle to obtain conformity. 


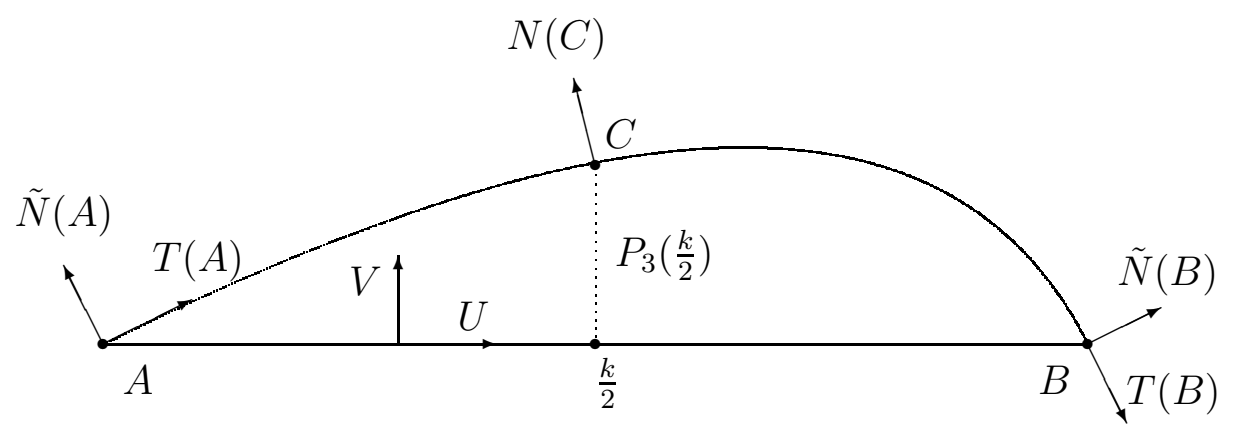

Fig. 5. Approximation of the middle surface in the plane $\Pi$. 


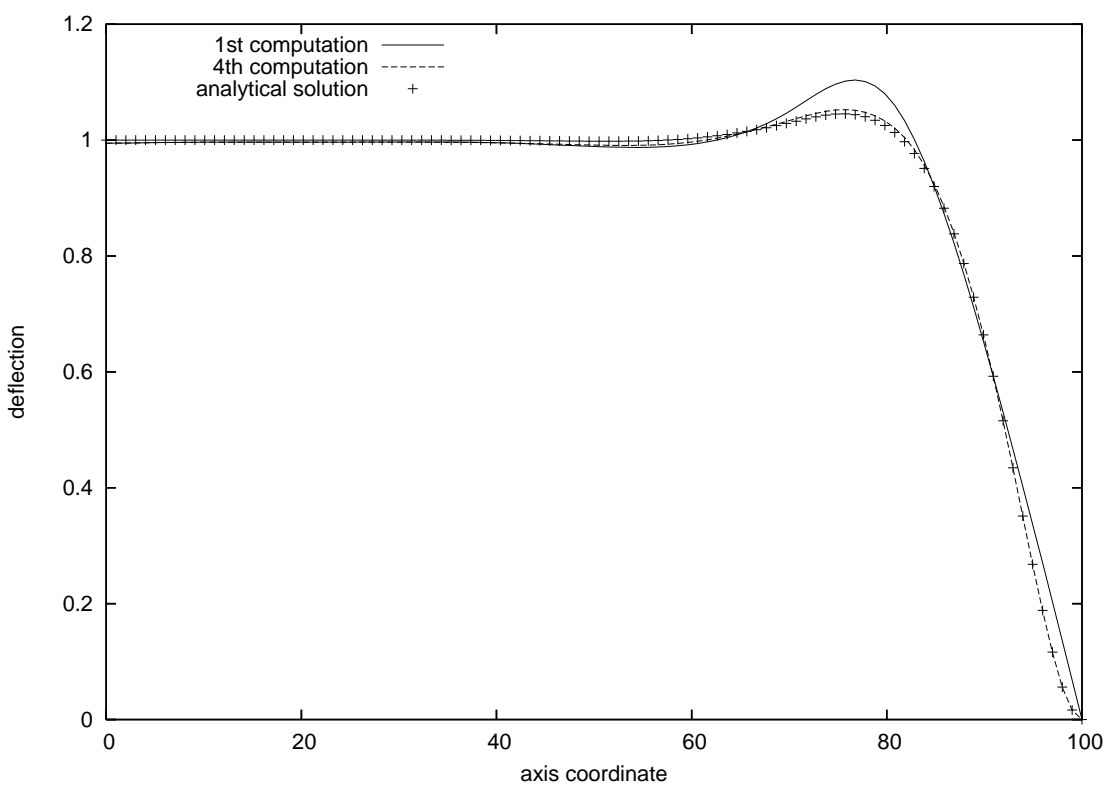

Fig. 6. Cylinder under uniform pressure $(\alpha=0.25)$ - Deflection along the axis direction. 


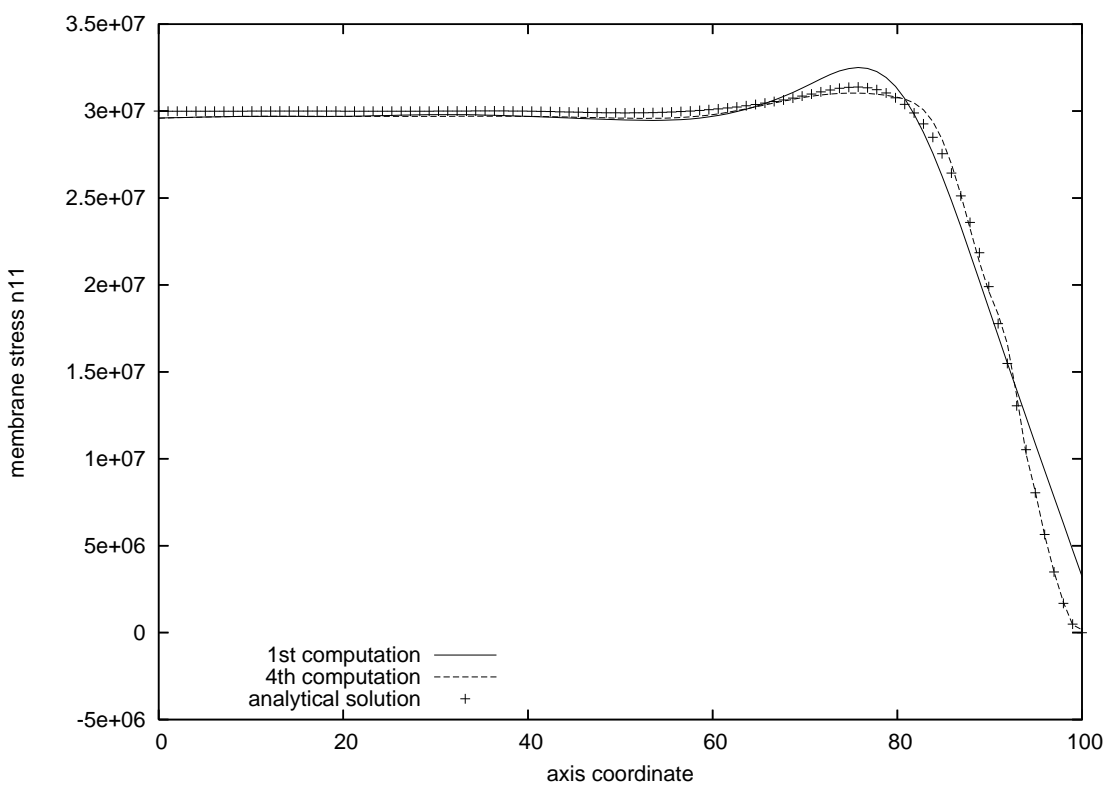

Fig. 7. Cylinder under uniform pressure $(\alpha=0.25)$ - Membrane stress $n_{1}^{1}$ along the axis direction. 


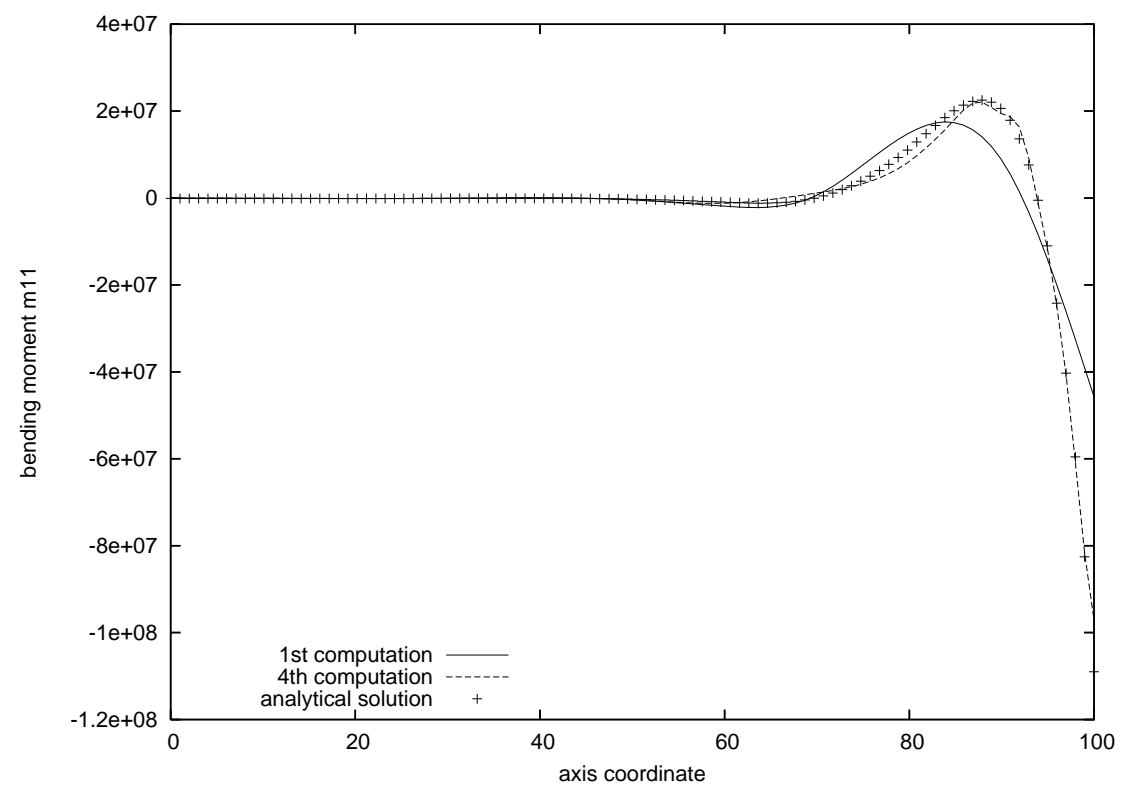

Fig. 8. Cylinder under uniform pressure $(\alpha=0.25)$ - Bending moment $m_{1}^{1}$ along the axis direction. 
56

57

58 along the axis direction. 


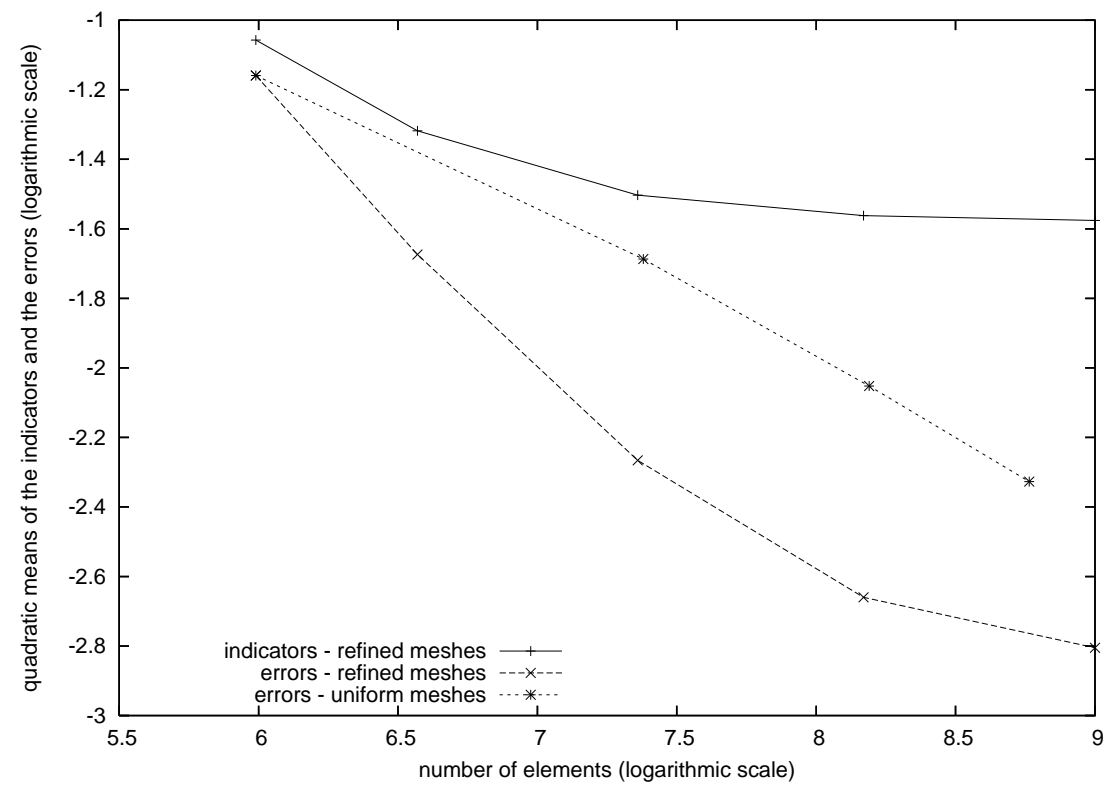

Fig. 10. Cylinder under uniform pressure $(\alpha=0)$ - Quadratic means of the error indicators and the "true" errors. 


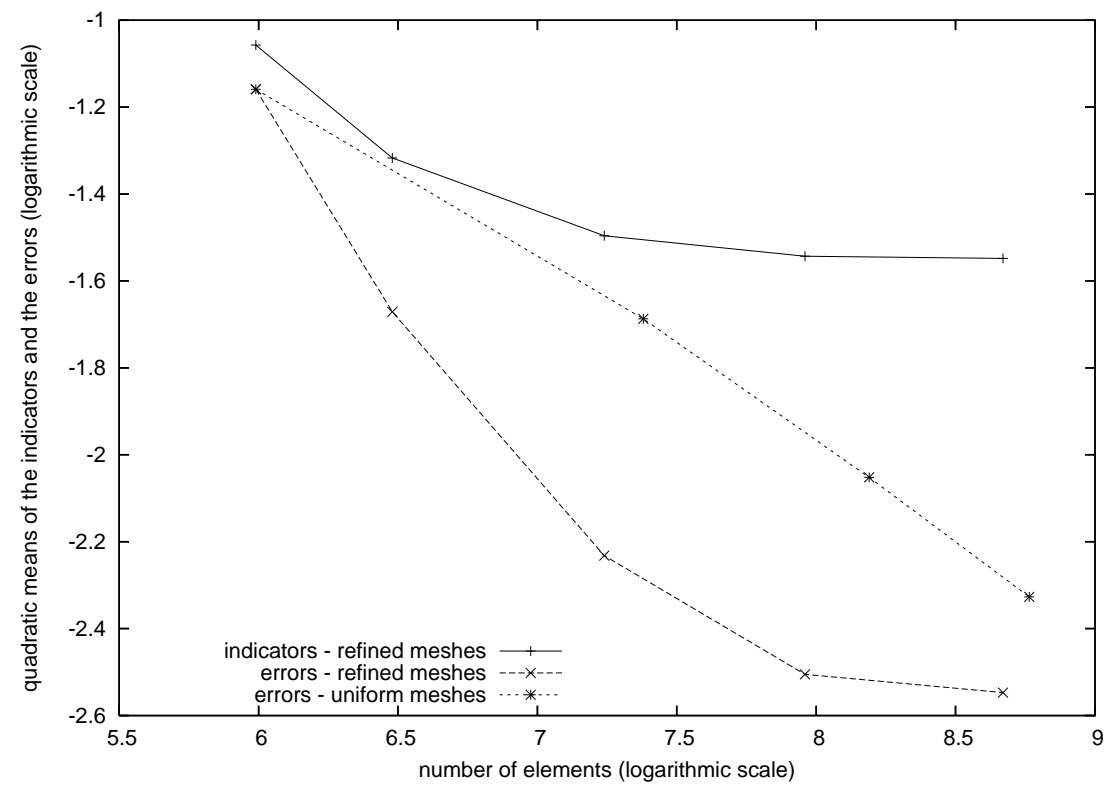

Fig. 11. Cylinder under uniform pressure $(\alpha=0.25)$ - Quadratic means of the error indicators and the "true" errors. 


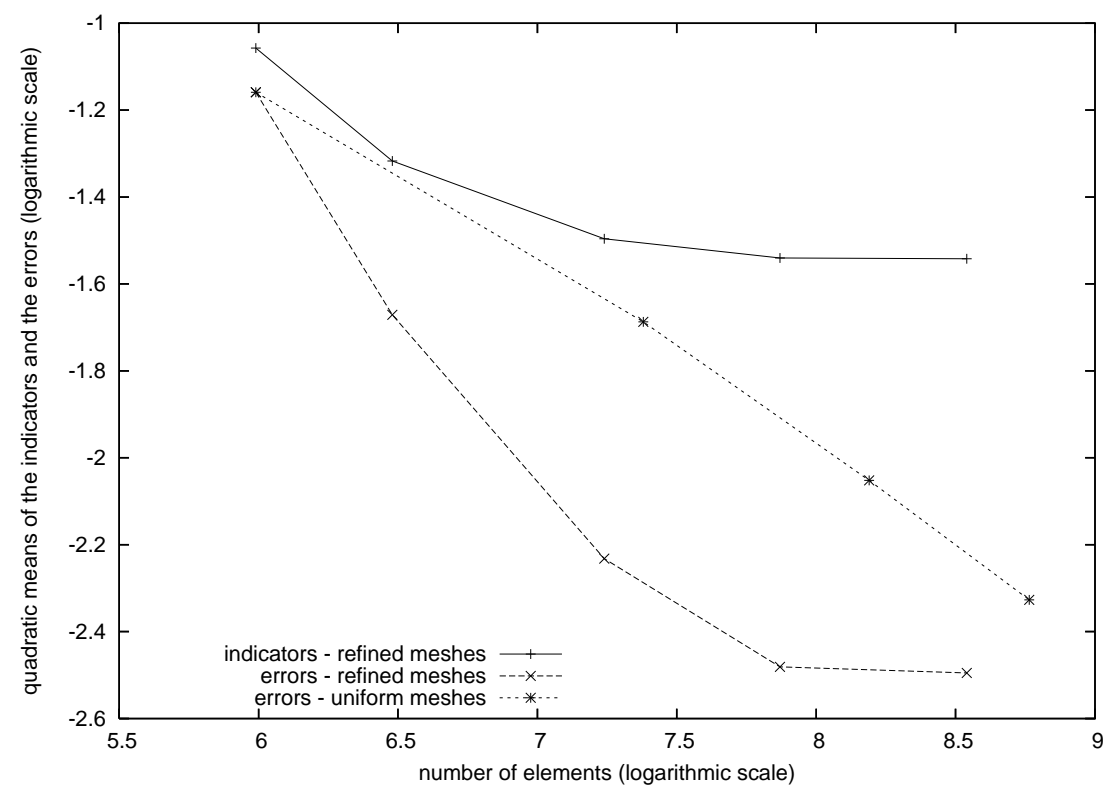

Fig. 12. Cylinder under uniform pressure $(\alpha=0.5)$ - Quadratic means of the error indicators and the "true" errors. 


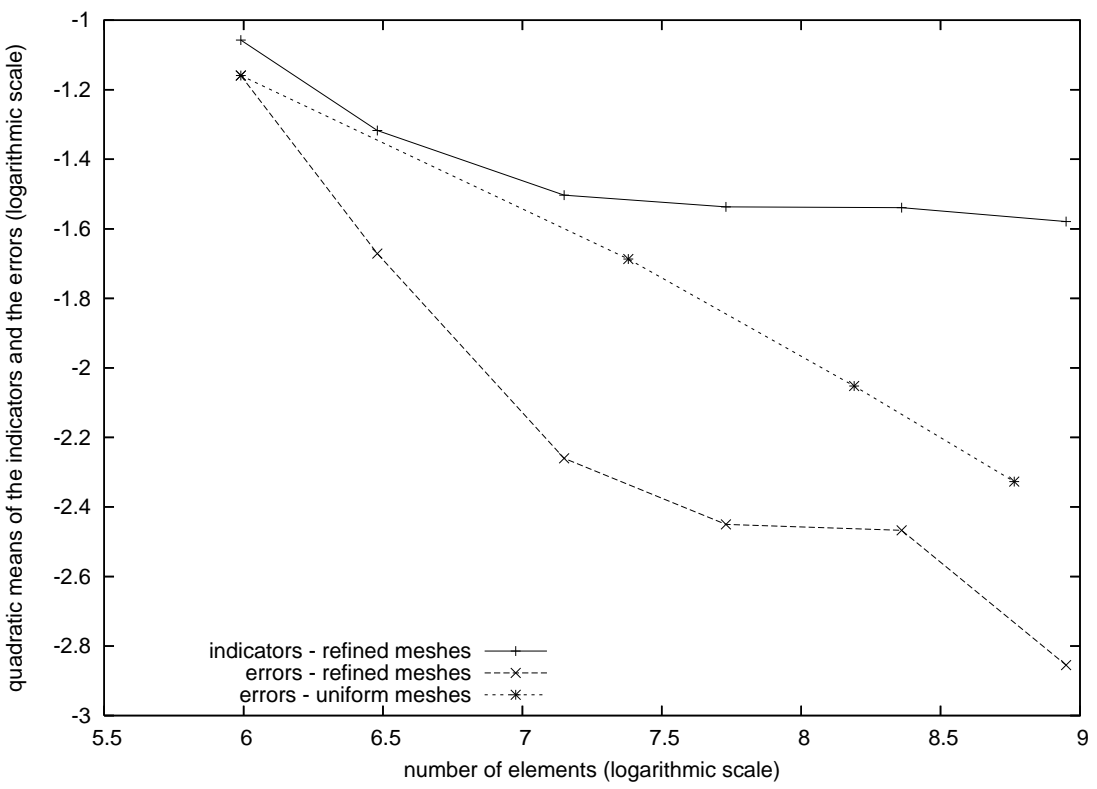

Fig. 13. Cylinder under uniform pressure $(\alpha=0.75)$ - Quadratic means of the error indicators and the "true" errors. 


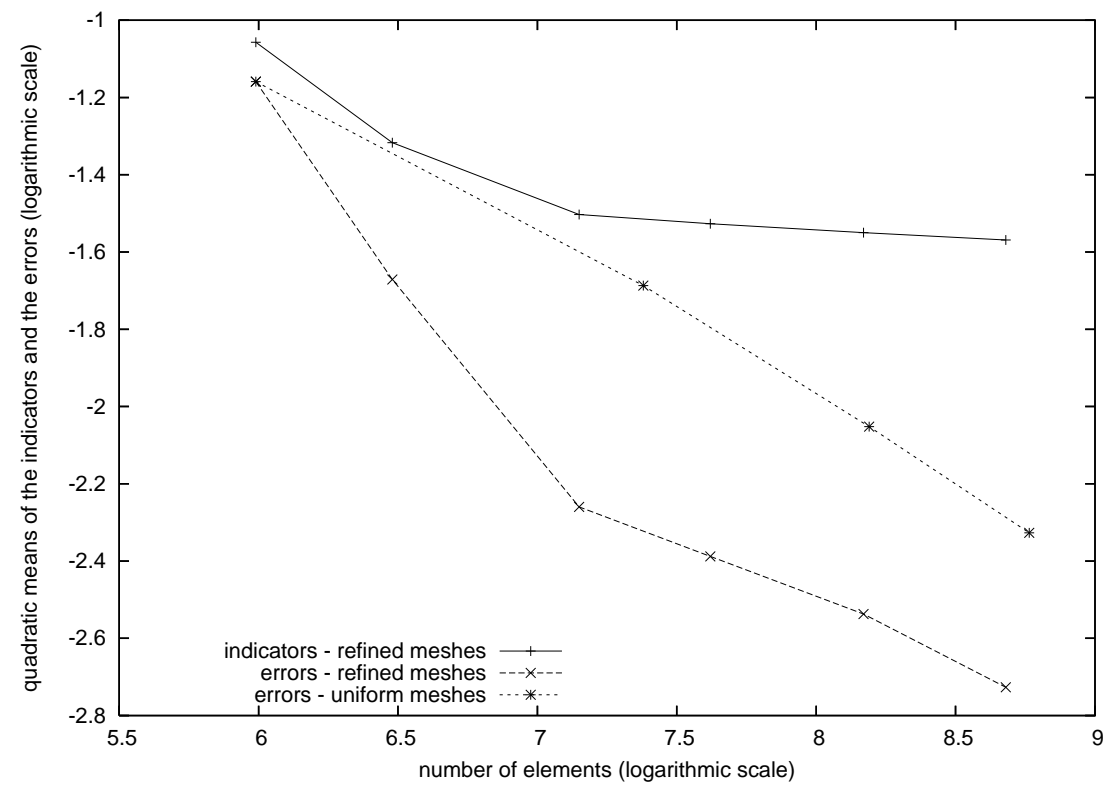

Fig. 14. Cylinder under uniform pressure $(\alpha=1)$ - Quadratic means of the error indicators and the "true" errors. 


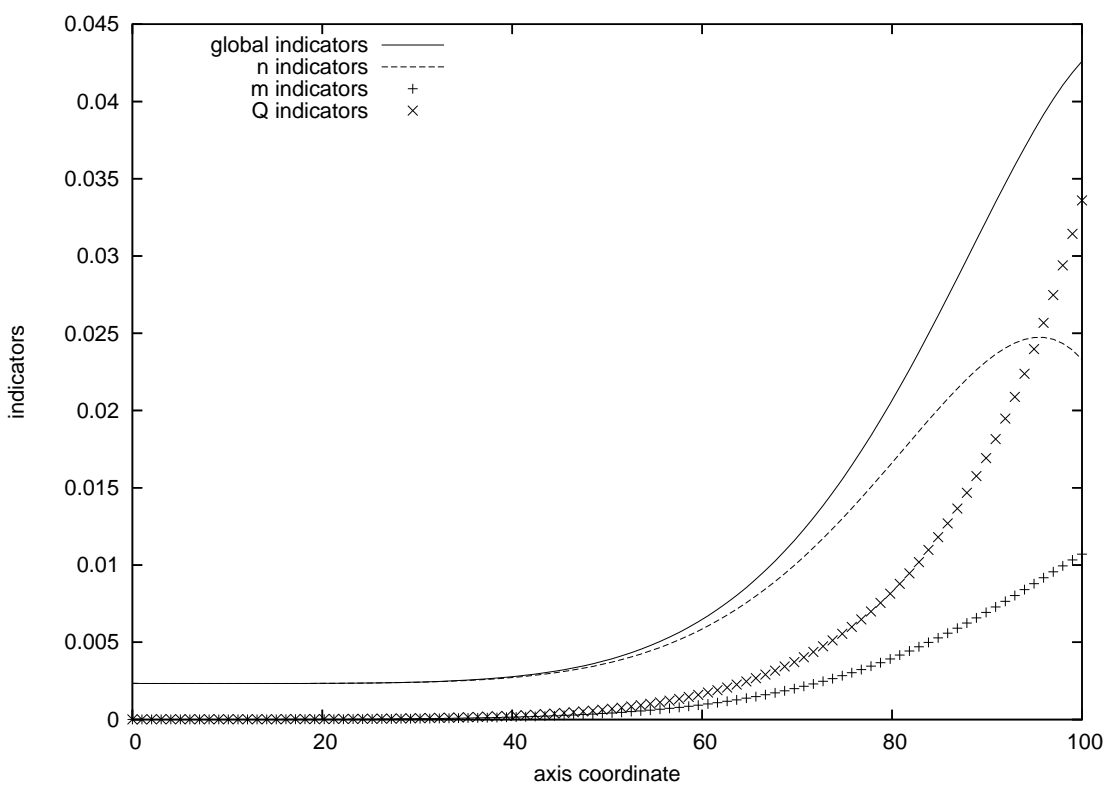

Fig. 15. Cylinder under uniform pressure - First computation - Error indicators $\eta$, $\eta_{n} / \sqrt{J_{0}}, \eta_{m} / \sqrt{J_{0}}$ and $\eta_{q} / \sqrt{J_{0}}$ along the axis direction. 


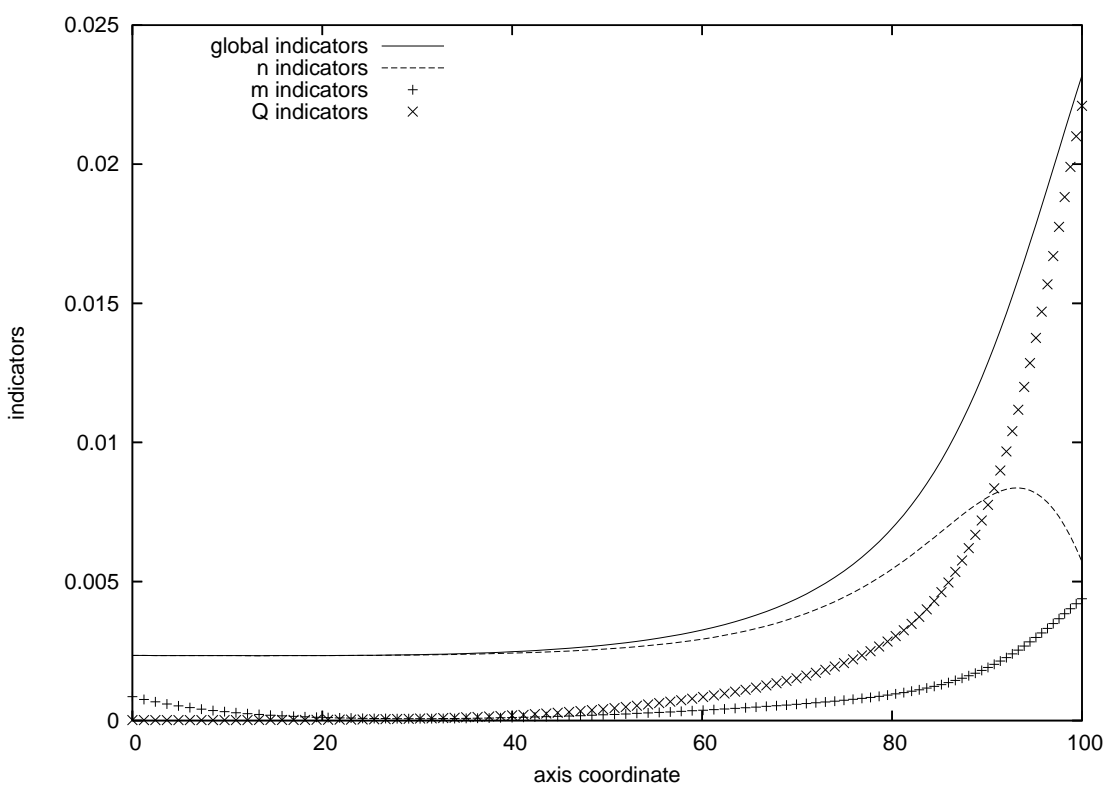

Fig. 16. Cylinder under uniform pressure - First refinement $(\alpha=0.25)$ - Error indicators $\eta, \eta_{n} / \sqrt{J_{0}}, \eta_{m} / \sqrt{J_{0}}$ and $\eta_{q} / \sqrt{J_{0}}$ along the axis direction. 


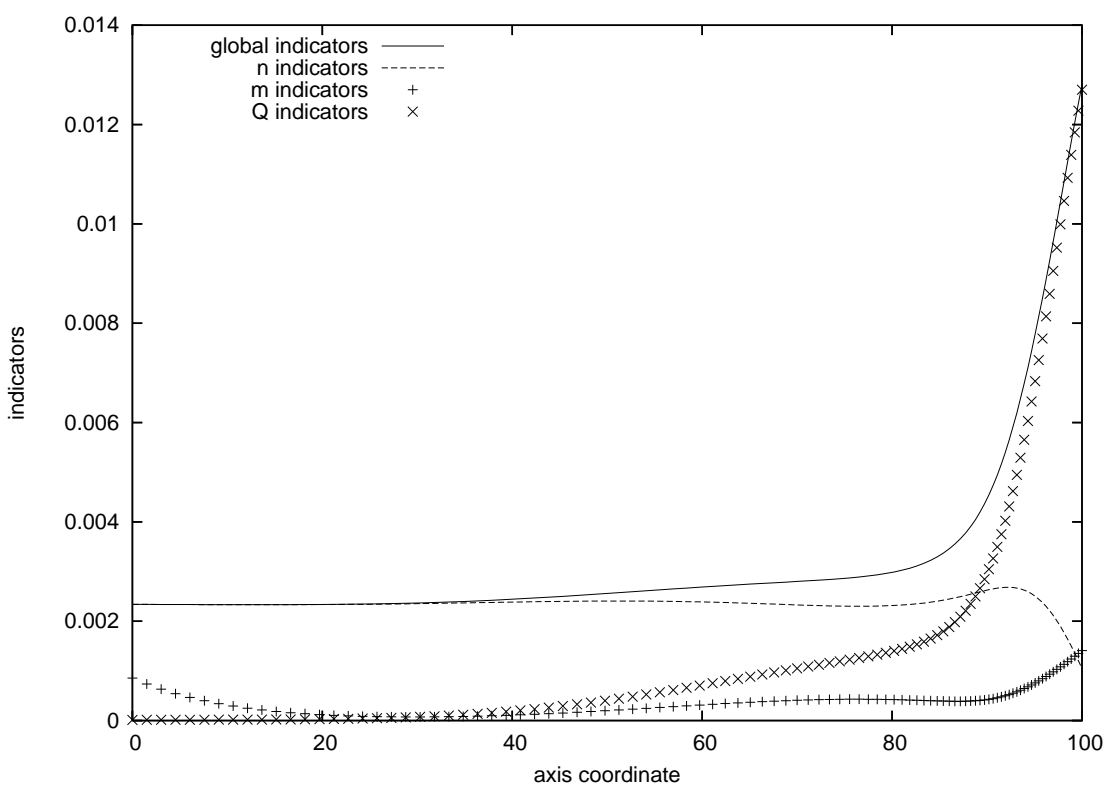

Fig. 17. Cylinder under uniform pressure - Second refinement $(\alpha=0.25)$ - Error indicators $\eta, \eta_{n} / \sqrt{J_{0}}, \eta_{m} / \sqrt{J_{0}}$ and $\eta_{q} / \sqrt{J_{0}}$ along the axis direction. 


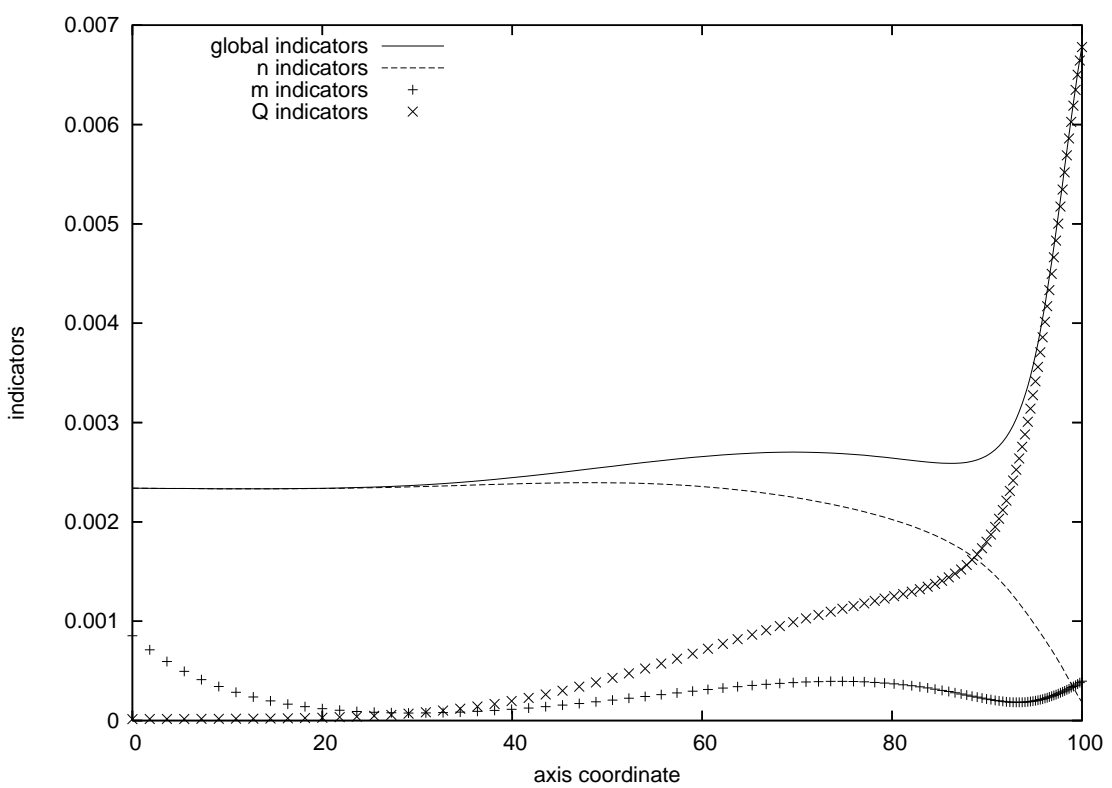

Fig. 18. Cylinder under uniform pressure - Third refinement $(\alpha=0.25)$ - Error indicators $\eta, \eta_{n} / \sqrt{J_{0}}, \eta_{m} / \sqrt{J_{0}}$ and $\eta_{q} / \sqrt{J_{0}}$ along the axis direction. 


$\begin{array}{ll}\text { length } & L=6 \mathrm{~m} \\ \text { thickness } & 2 \varepsilon=0.03 \mathrm{~m} \\ \text { radius } & R=3 \mathrm{~m} \\ \text { Young's modulus } & E=3 \times 10^{4} \mathrm{MPa} \\ \text { Poisson's ratio } & \nu=0.3 \\ \text { force } & F=1 \mathrm{~N}\end{array}$

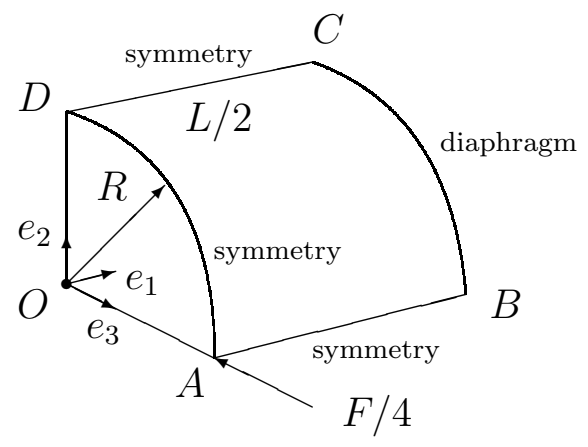

Fig. 19. Settings for the pinched cylinder. 

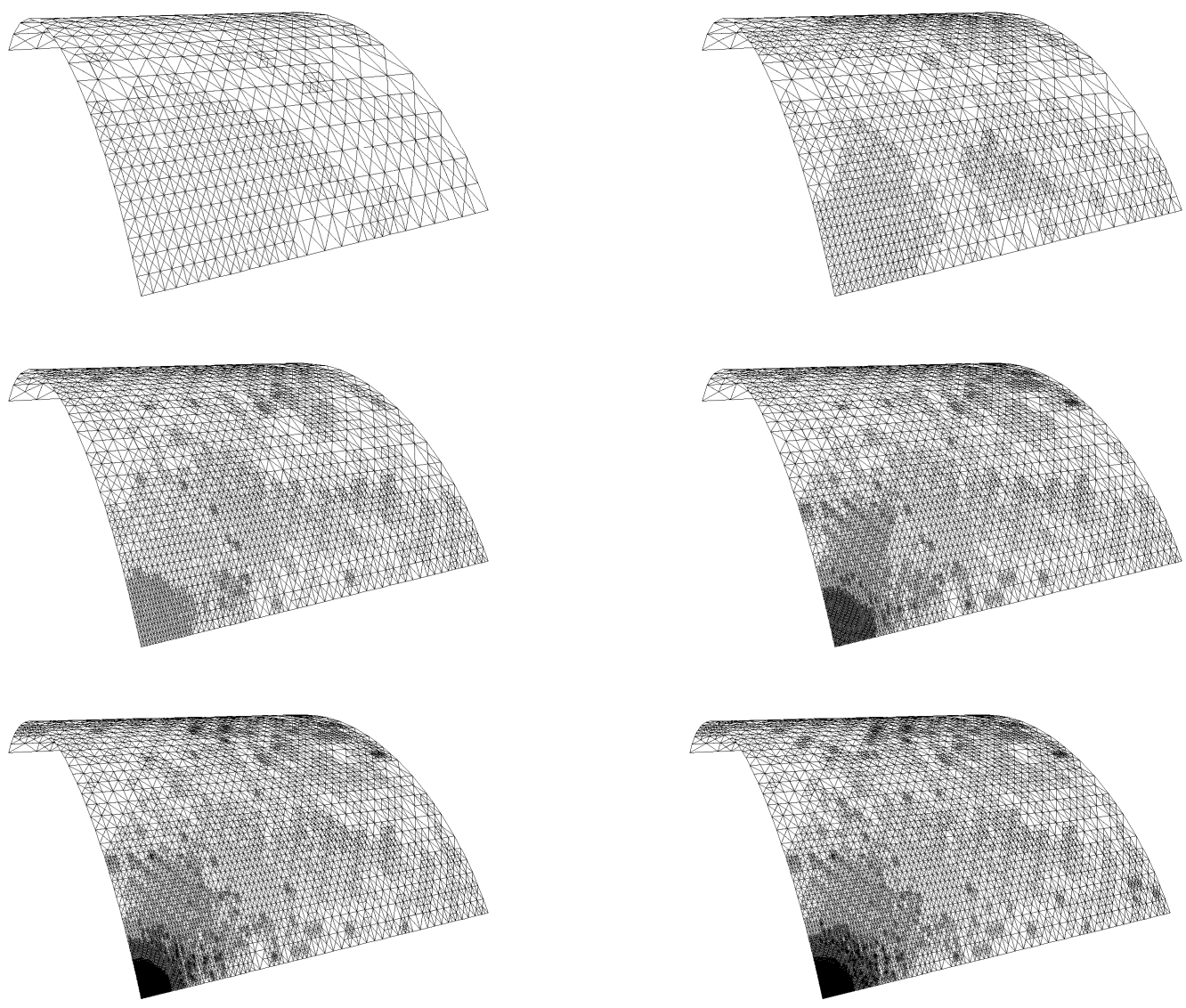

Fig. 20. Successive refinements of the pinched cylinder $-\alpha=0.25$. 
50

51

52

53

54

55

56

57

58

59

60

61

62
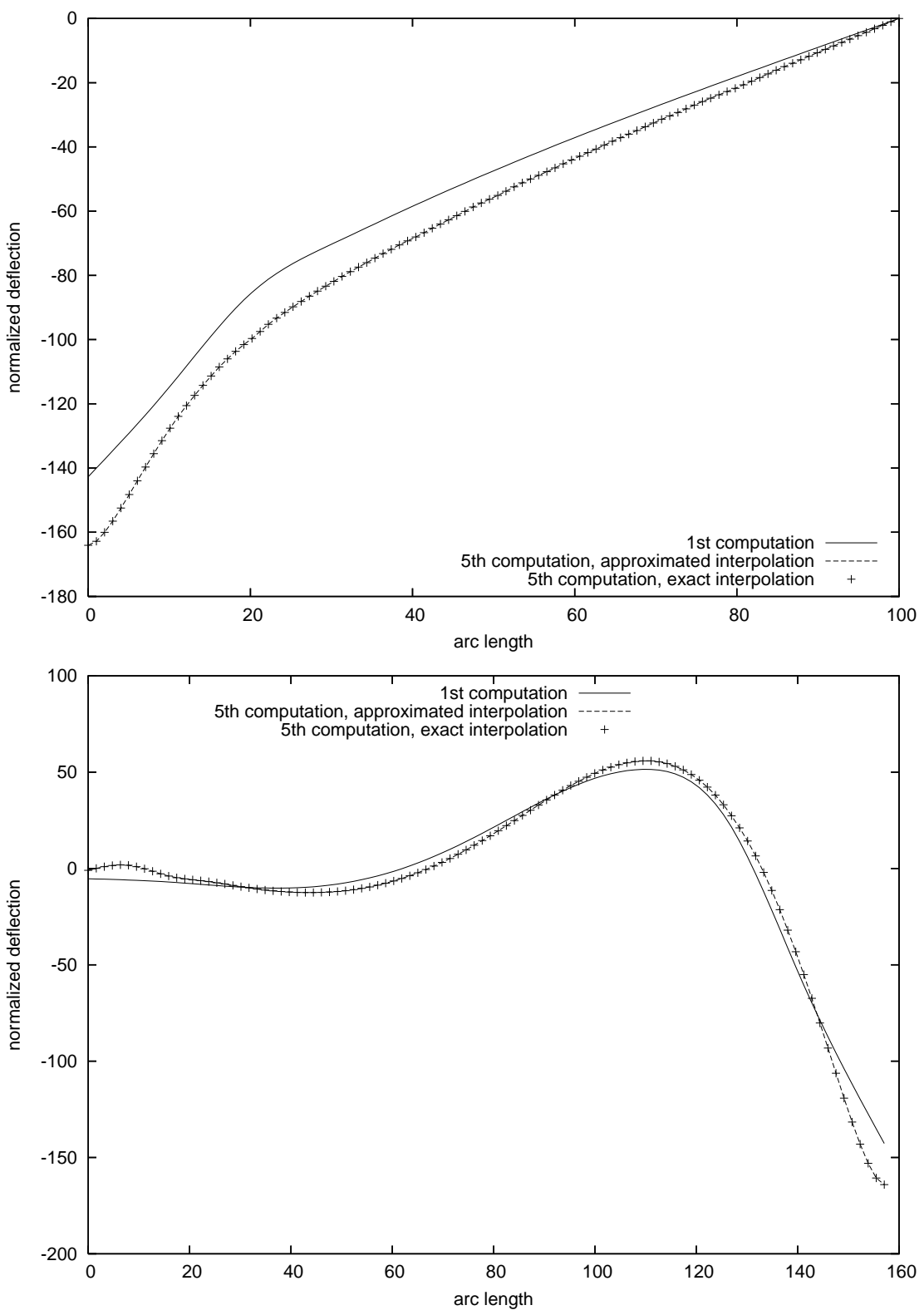

Fig. 21. Pinched cylinder $(\alpha=0.25)$ - Normalized deflection along $\widehat{A B}$ (top) and $\widehat{A D}$ (bottom). 
50

51

52

53

54

55

56

57

58

59

60

61

62

63

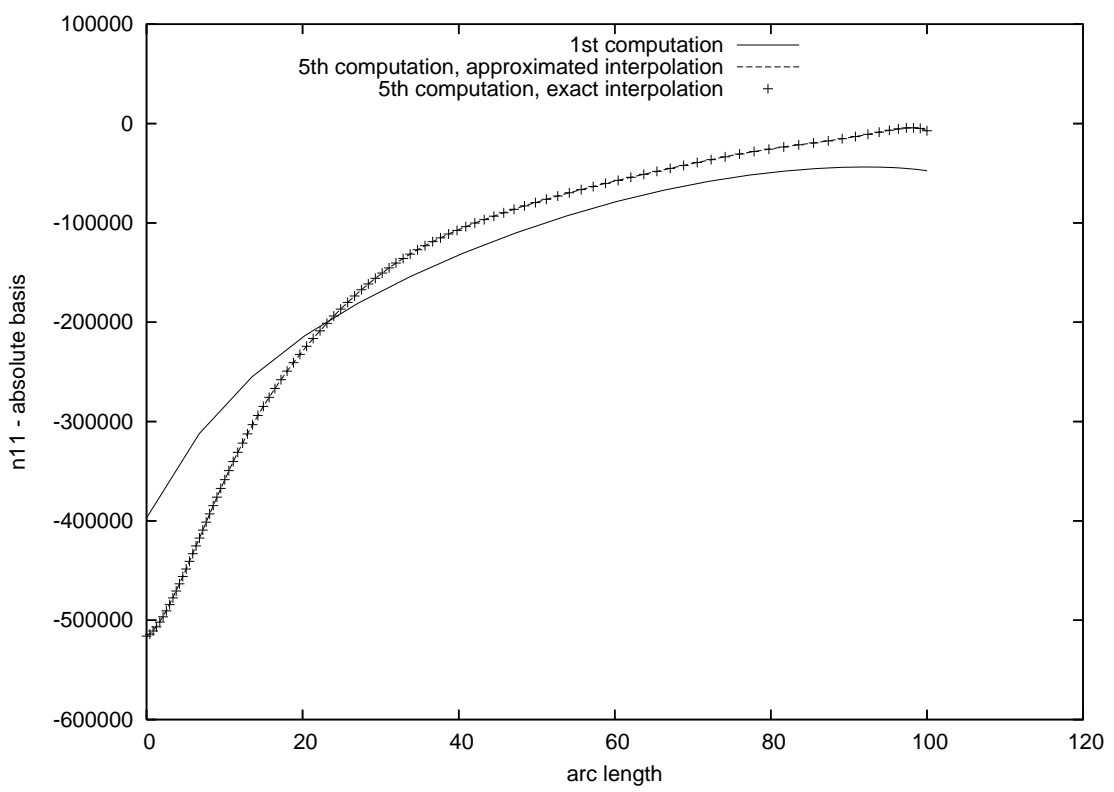

Fig. 22. Pinched cylinder $(\alpha=0.25)-n_{11}$ component in the absolute basis of the resultant stresses along $\overparen{A B}$. 


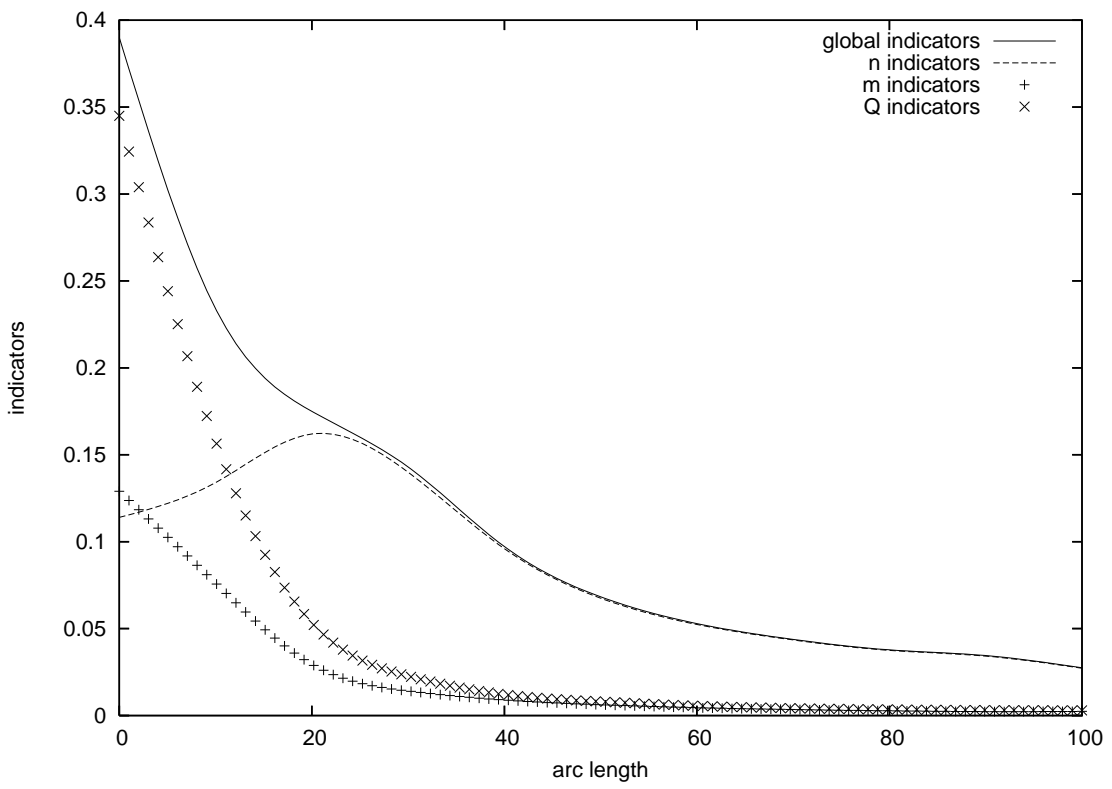

Fig. 23. Pinched cylinder - First computation - Error indicators $\eta, \eta_{n} / \sqrt{J_{0}}, \eta_{m} / \sqrt{J_{0}}$ and $\eta_{q} / \sqrt{J_{0}}$ along $\overparen{A B}$. 


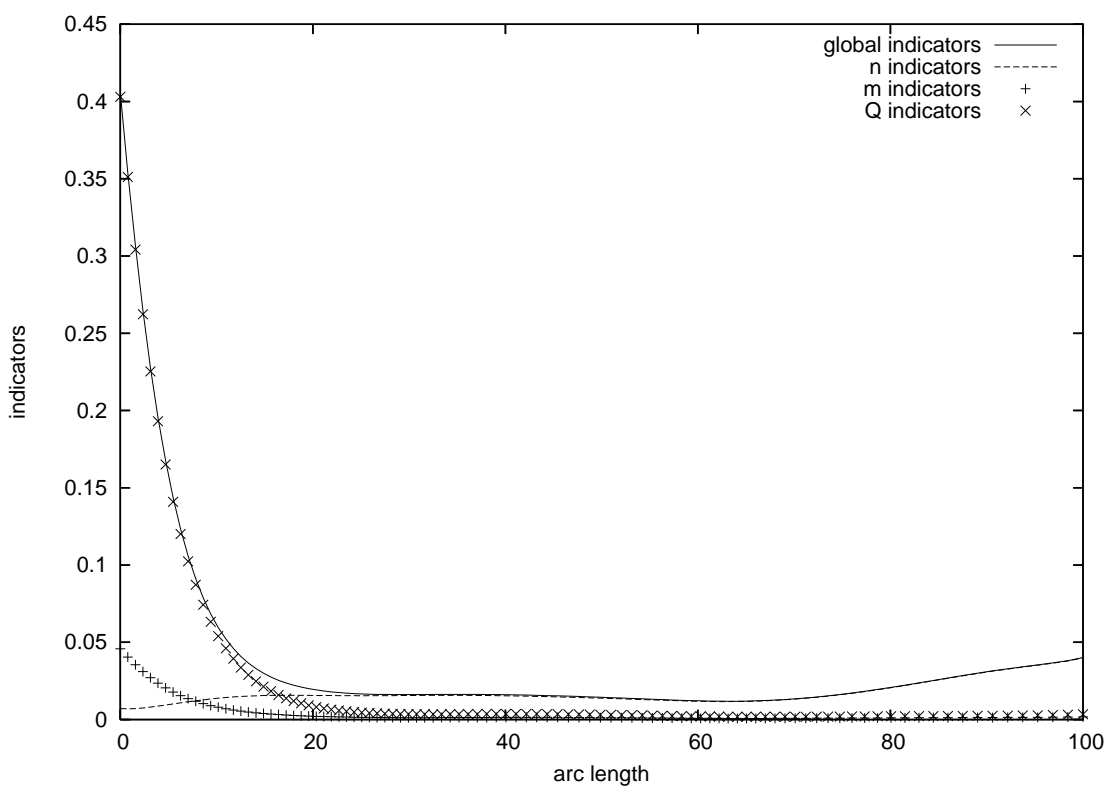

Fig. 24. Pinched cylinder - Second refinement $(\alpha=0.25)$ - Error indicators $\eta$, $\eta_{n} / \sqrt{J_{0}}, \eta_{m} / \sqrt{J_{0}}$ and $\eta_{q} / \sqrt{J_{0}}$ along $\overparen{A B}$. 


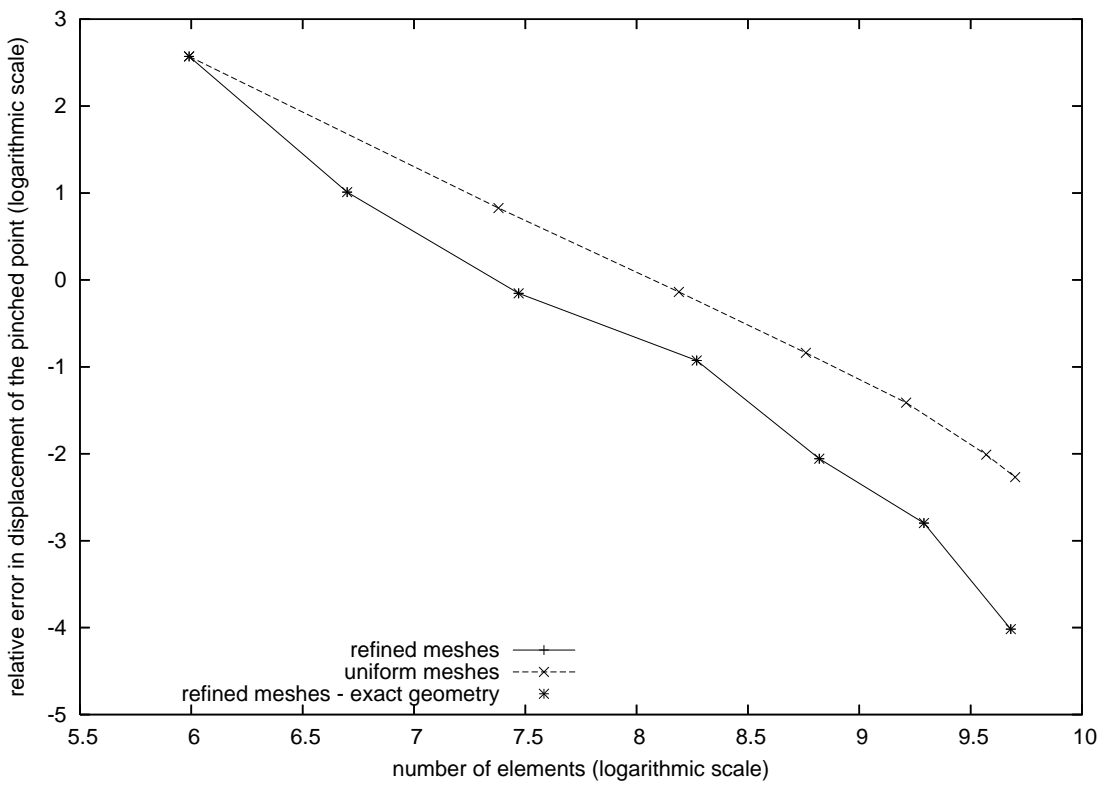

Fig. 25. Pinched cylinder $(\alpha=0.25)$ - Relative error $(\%)$ of the displacement of the pinched point, in logarithmic scale (Reference value: -164.24) - Refined and uniform meshes with exact geometry. 


\begin{tabular}{|ll|}
\hline length & $L=12 \mathrm{~m}$ \\
thickness & $2 \varepsilon=0.32 \mathrm{~m}$ \\
width & $b=1.1 \mathrm{~m}$ \\
Young's modulus & $E=29 \mathrm{MPa}$ \\
Poisson's ratio & $\nu=0.22$ \\
force & $F=1 \mathrm{~N}$ \\
\hline
\end{tabular}

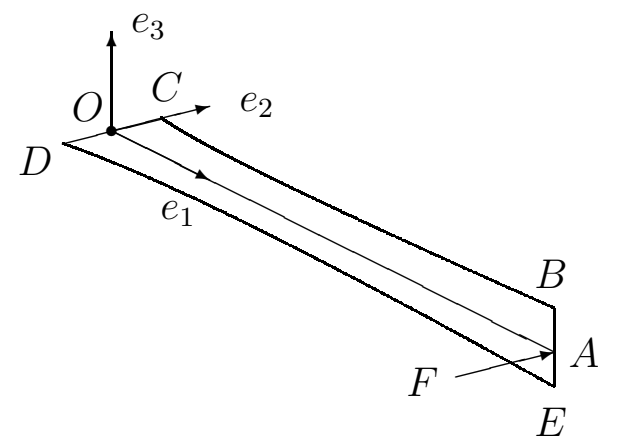

Fig. 26. Settings for the twisted ribbon. 


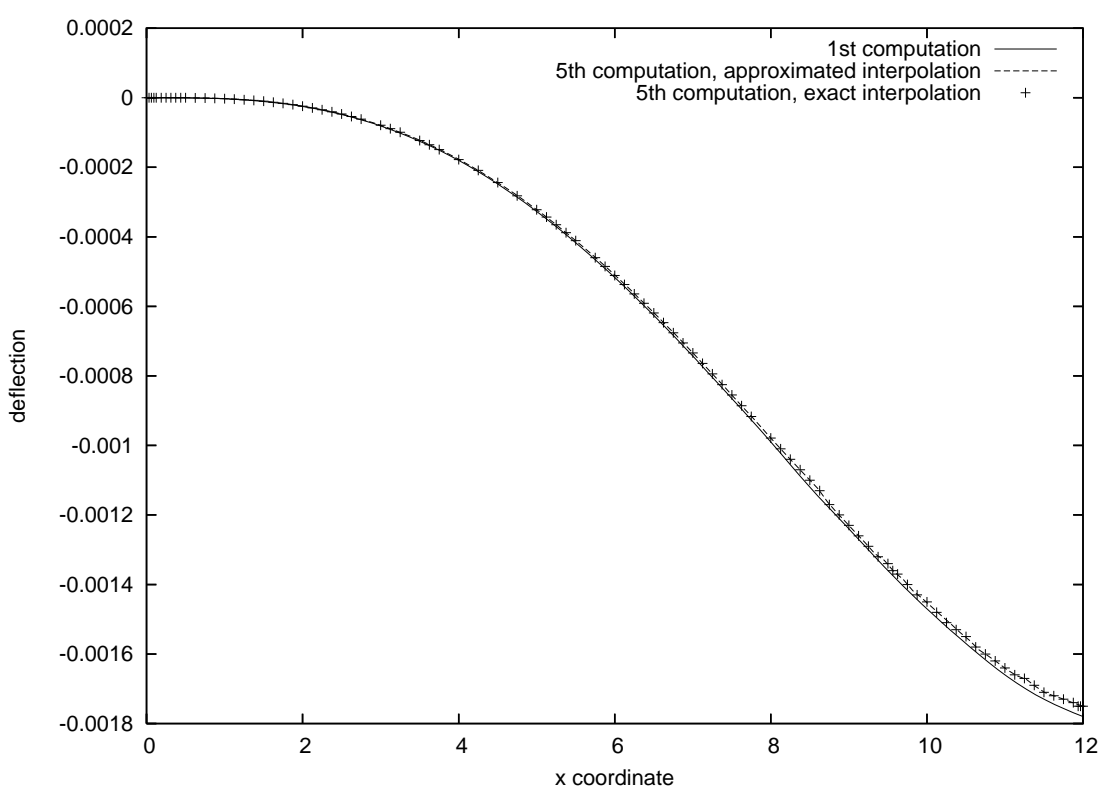

Fig. 27. Twisted ribbon $(\alpha=0.5)$ - Deflection along $\overparen{D E}$. 


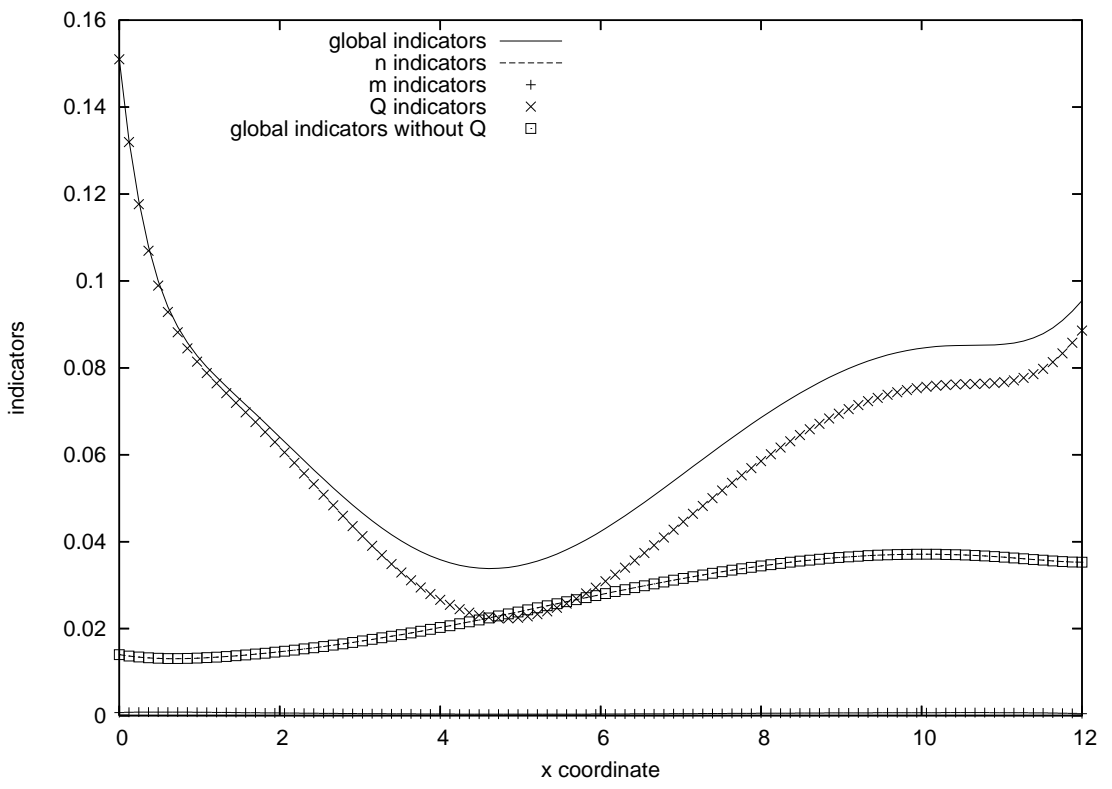

Fig. 28. Twisted ribbon - First computation. Error indicators $\eta, \eta_{n} / \sqrt{J_{0}}, \eta_{m} / \sqrt{J_{0}}$ and $\eta_{q} / \sqrt{J_{0}}$ along $\overparen{D E}$. The global indicators computed without the contribution of $\eta_{q} / \sqrt{J_{0}}$ are also presented. 


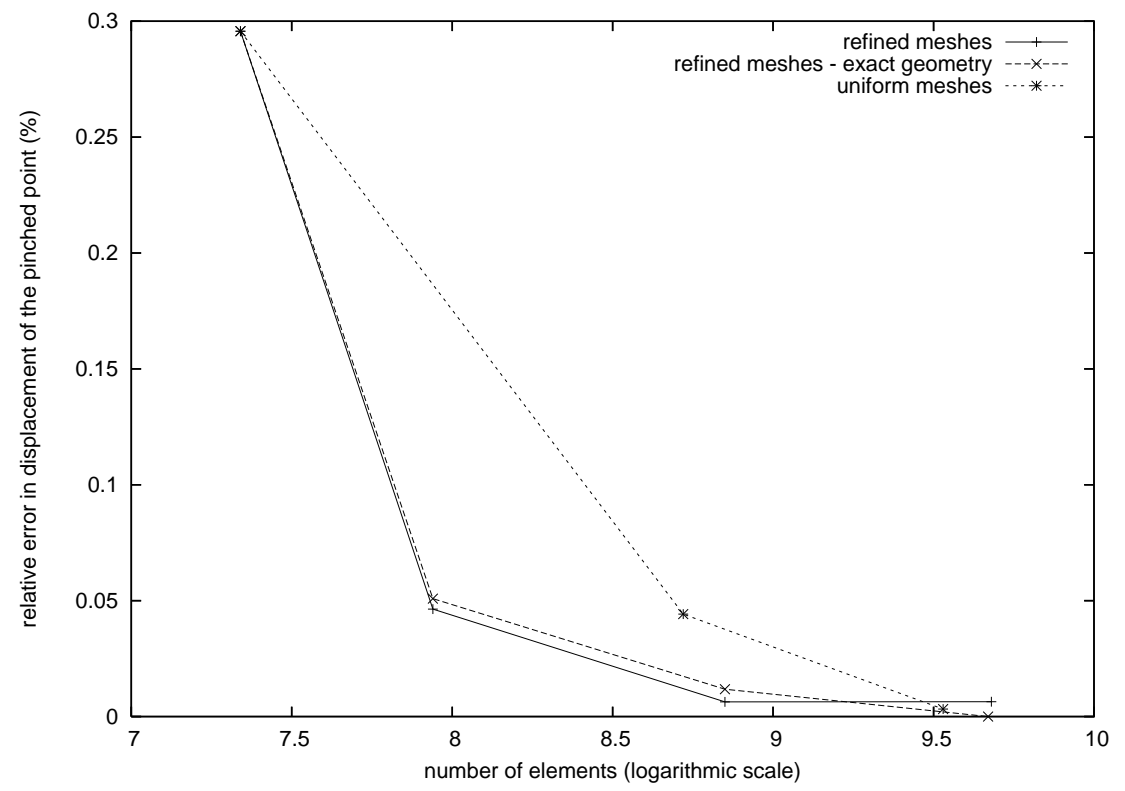

Fig. 29. Twisted ribbon $(\alpha=0.5)$ - Relative error (\%) of the displacement of the pinched point (Reference value: $1.7493 \times 10^{-3}$ ) - Refined and uniform meshes with exact geometry. 

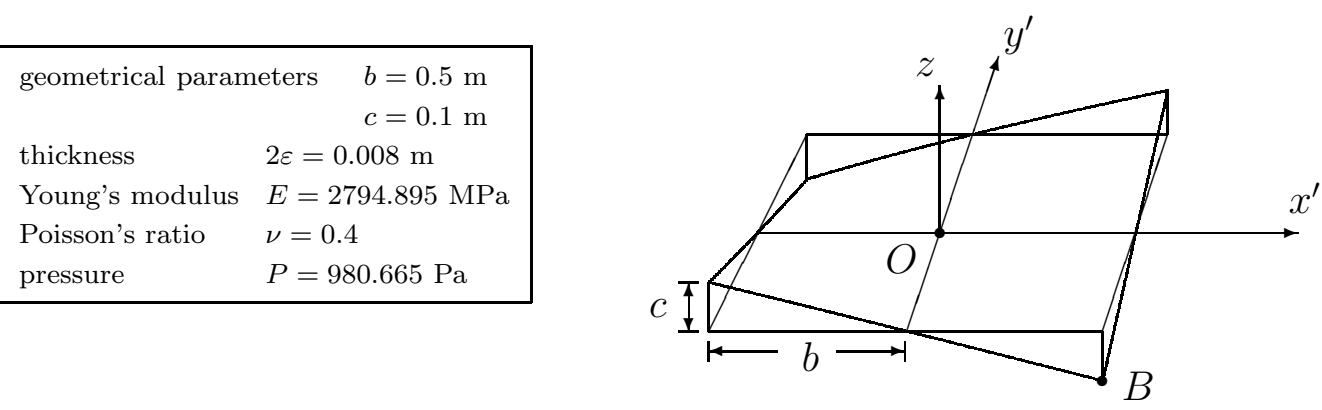

Fig. 30. Settings for the hyperbolic paraboloid. 


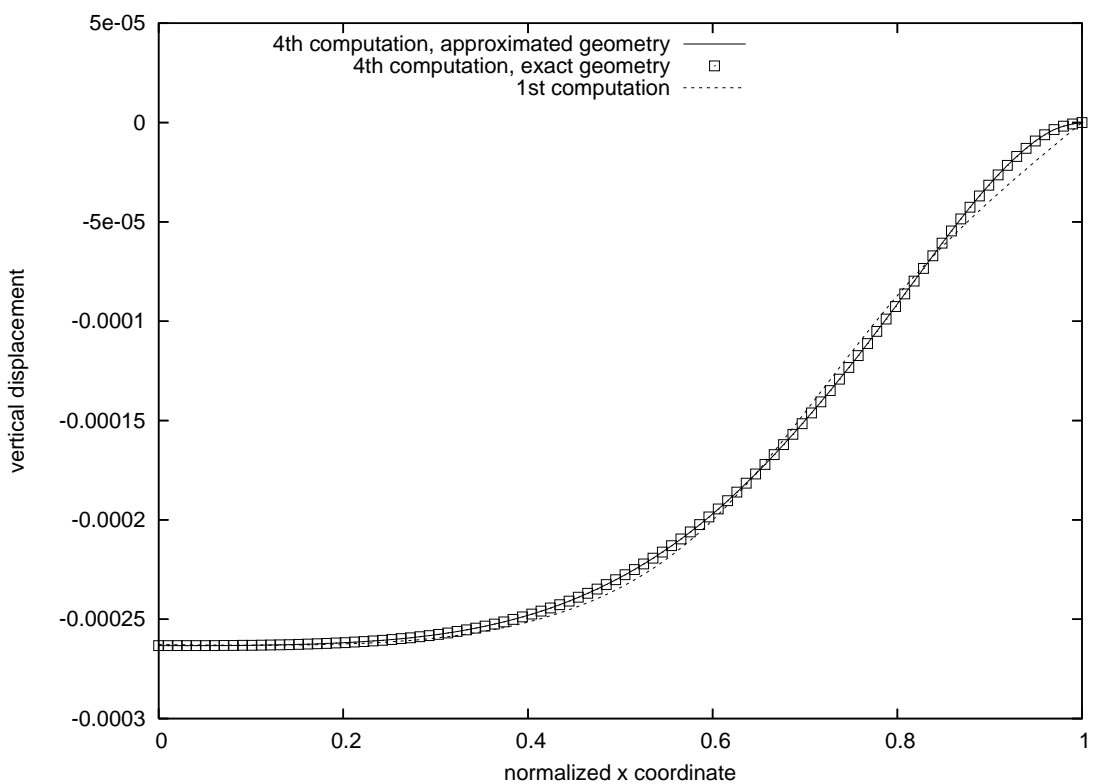

Fig. 31. Hyperbolic paraboloid - Vertical displacement of $\overparen{O B}$. 


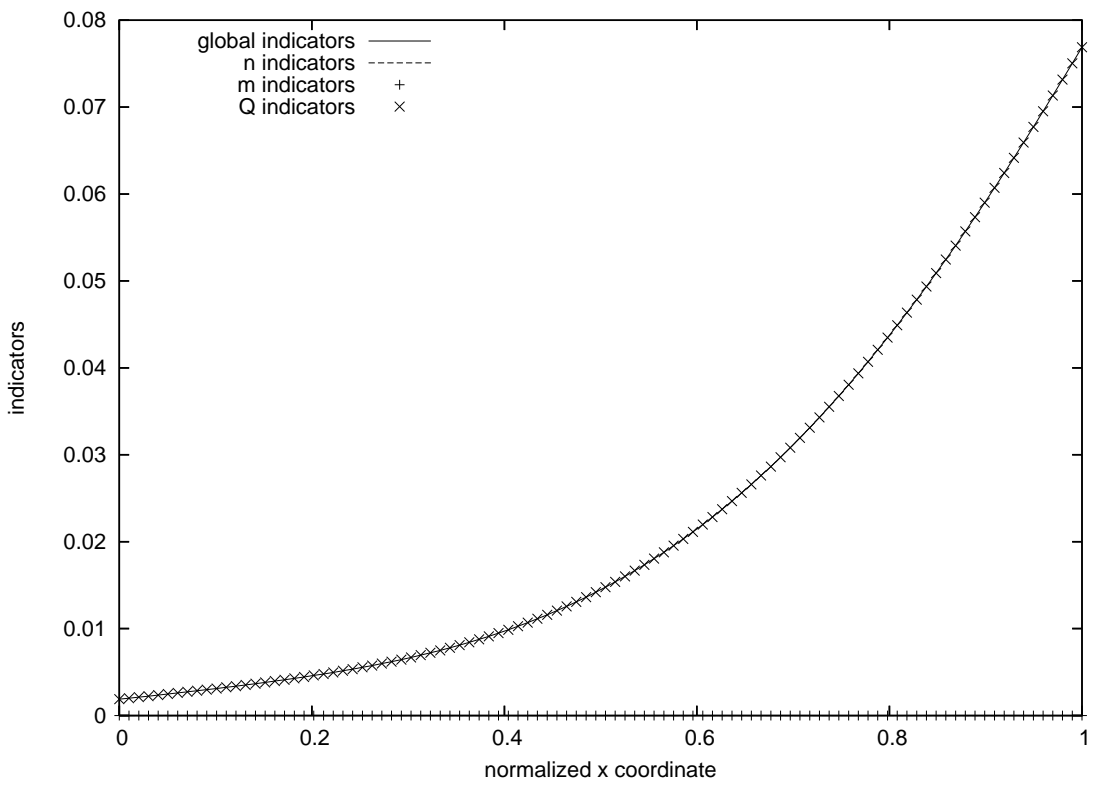

Fig. 32. Hyperbolic paraboloid - First computation - Error indicators $\eta, \eta_{n} / \sqrt{J_{0}}$, $\eta_{m} / \sqrt{J_{0}}$ and $\eta_{q} / \sqrt{J_{0}}$ along $\stackrel{O B}{O}$. 


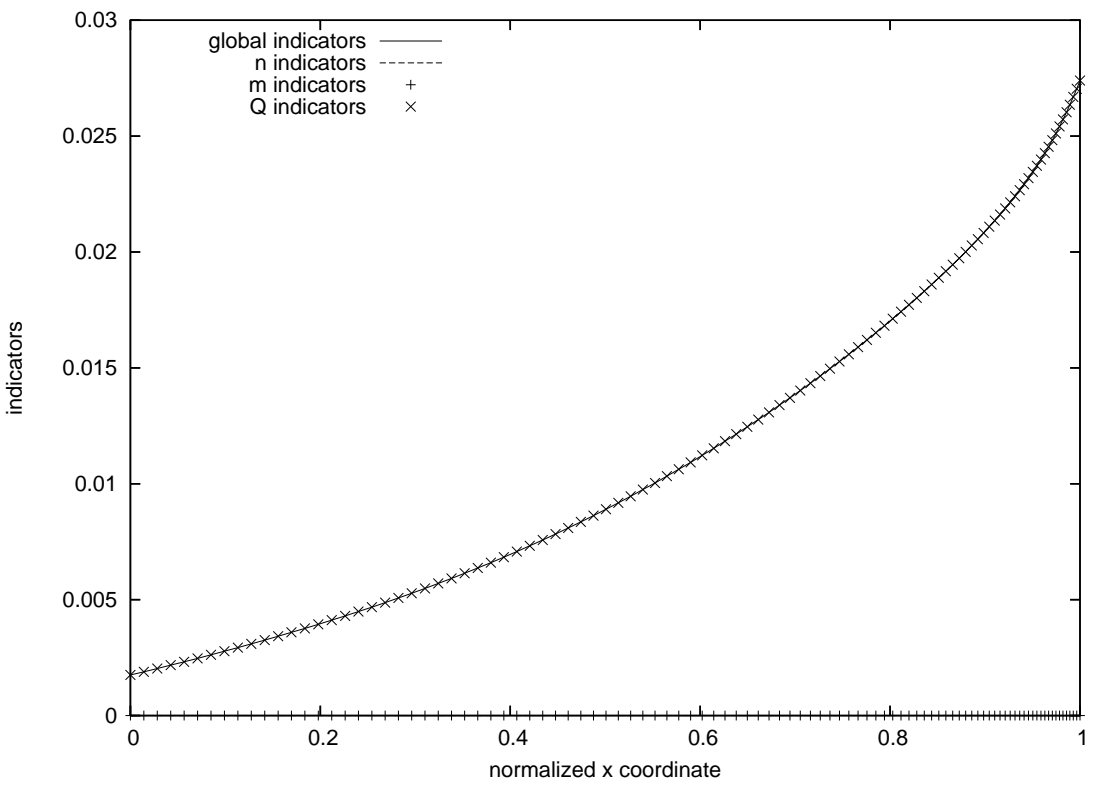

Fig. 33. Hyperbolic paraboloid - Second refinement $(\alpha=0)$ - Error indicators $\eta$, $\eta_{n} / \sqrt{J_{0}}, \eta_{m} / \sqrt{J_{0}}$ and $\eta_{q} / \sqrt{J_{0}}$ along $\overparen{O B}$. 


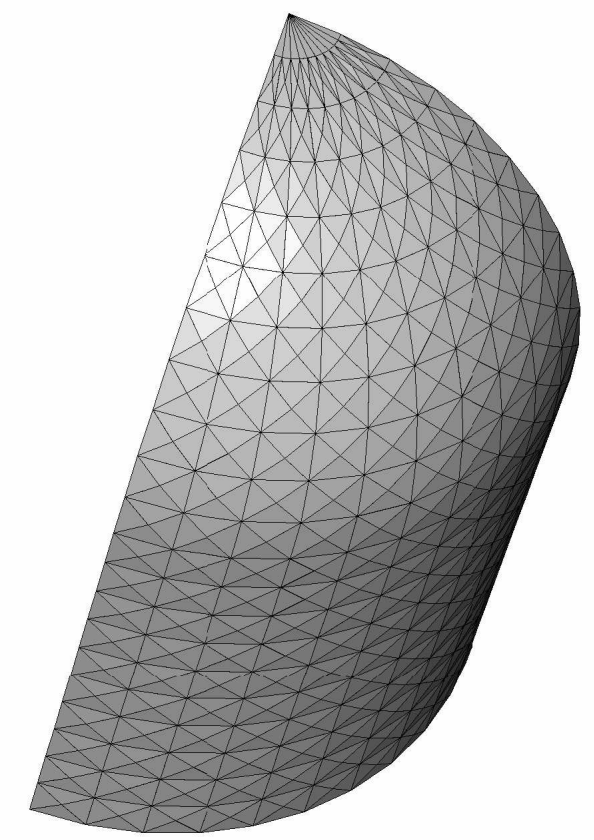

Fig. 34. Tank under uniform internal pressure - One eighth of the structure. 


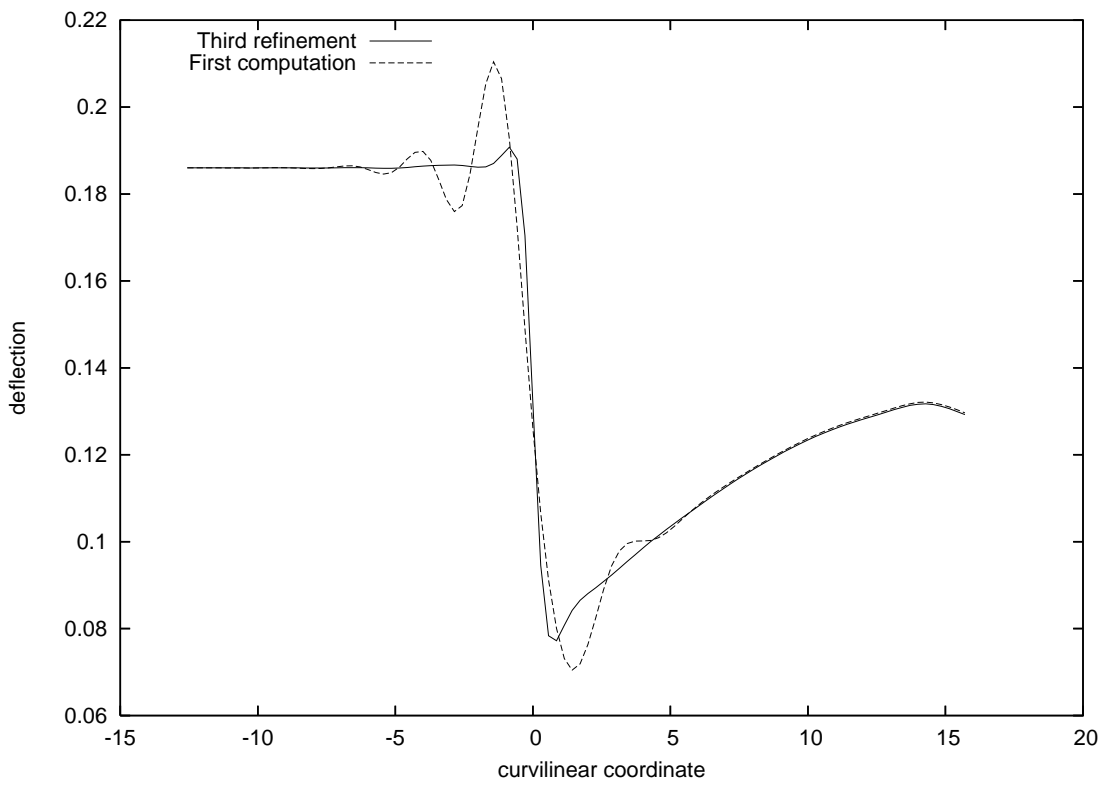

Fig. 35. Tank under uniform internal pressure - Deflection along the axis direction of the computational domain. The coordinates of the junction and the hemisphere top are 0.0 and 15.8 respectively. 

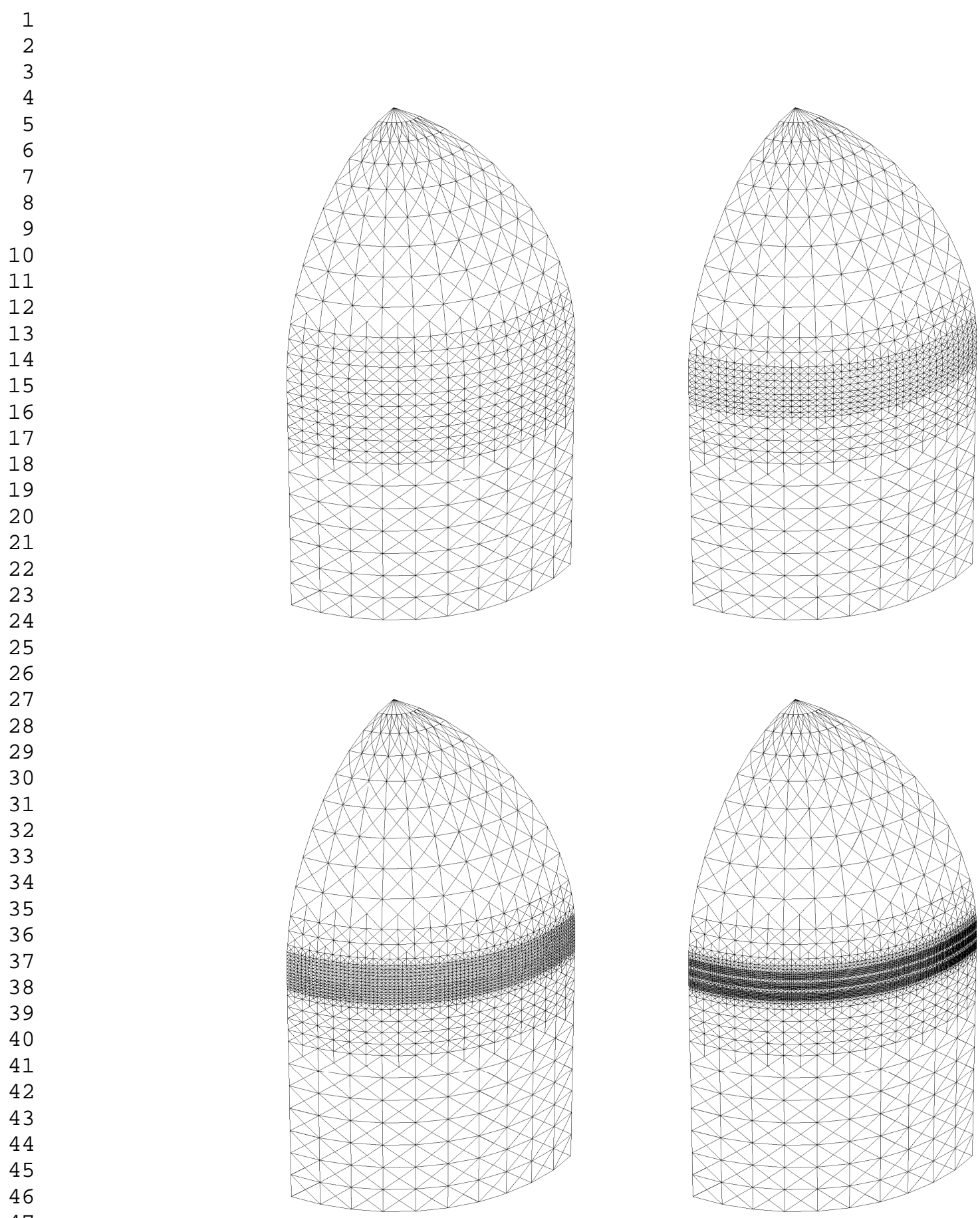

Fig. 36. Tank under uniform internal pressure $(\alpha=0)$ - Successive refinements. 


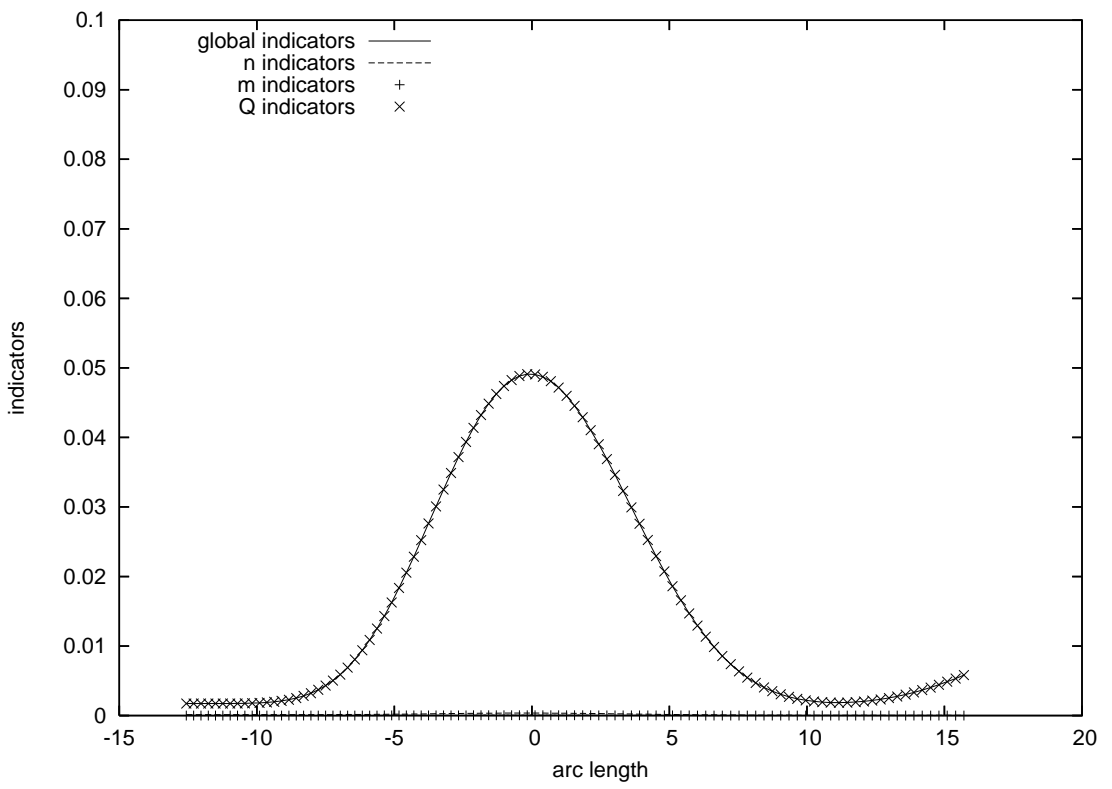

Fig. 37. Tank under uniform internal pressure - First computation. Error indicators $\eta, \eta_{n} / \sqrt{J_{0}}, \eta_{m} / \sqrt{J_{0}}$ and $\eta_{q} / \sqrt{J_{0}}$ along the axis direction of the computational domain. 


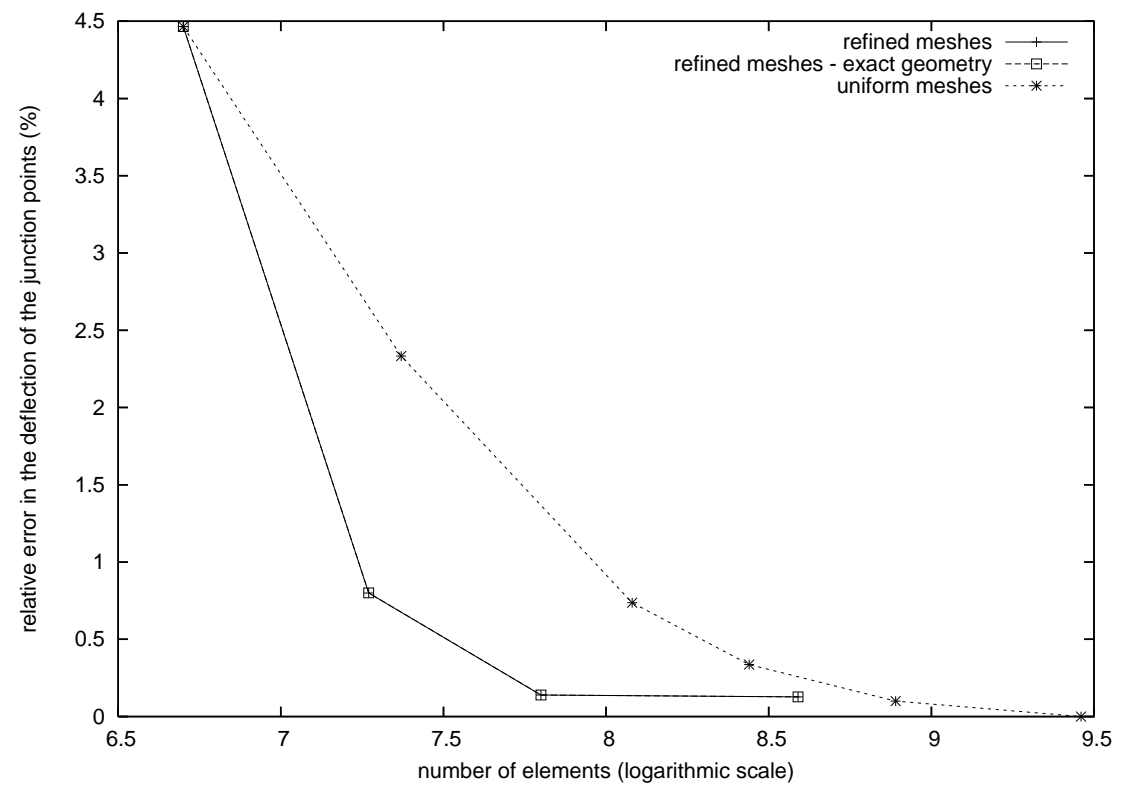

Fig. 38. Tank under uniform internal pressure $(\alpha=0)$ - Relative error (\%) of the deflection of the junction points (Reference value: $0.1319 \mathrm{~m}$ ) - Refined and uniform meshes with exact geometry. 


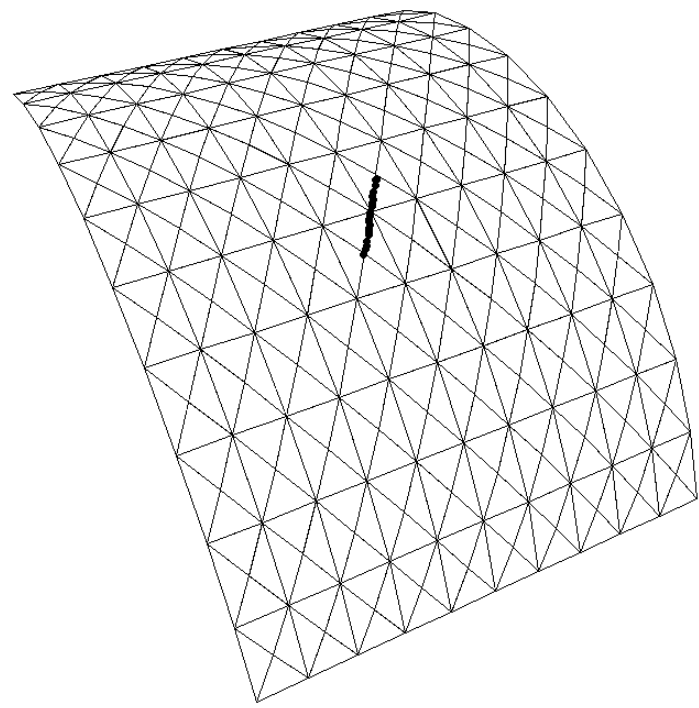

Fig. 39. Cracked cylindrical shell - Location of the crack on the initial mesh. 
2

3

4

5

6

7

8

10

11

12

13

14

15

16

17

18

19

20

21

22

23

24

25

26

27

28

29

30

31

32

33

34

35

36

37

38

39

40

41

42

43

44

45

46

47

48

49

50

51

52

53

54

55

56

57

58

59

60

61

62

63

64

65
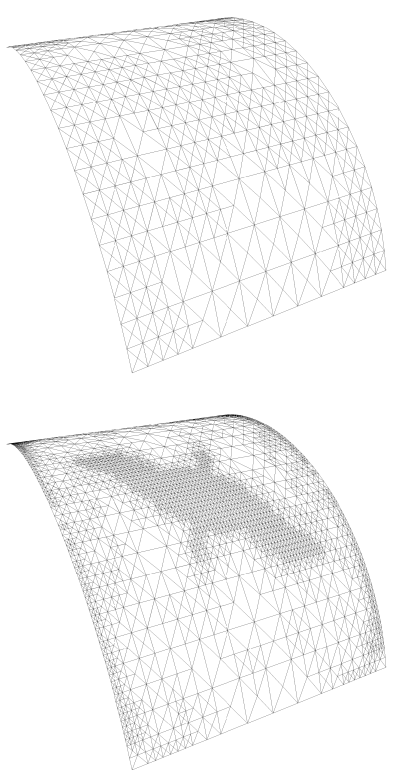

Fig. 40. Cracked cylindrical shell $(\alpha=0)$ - Successive refinements. 


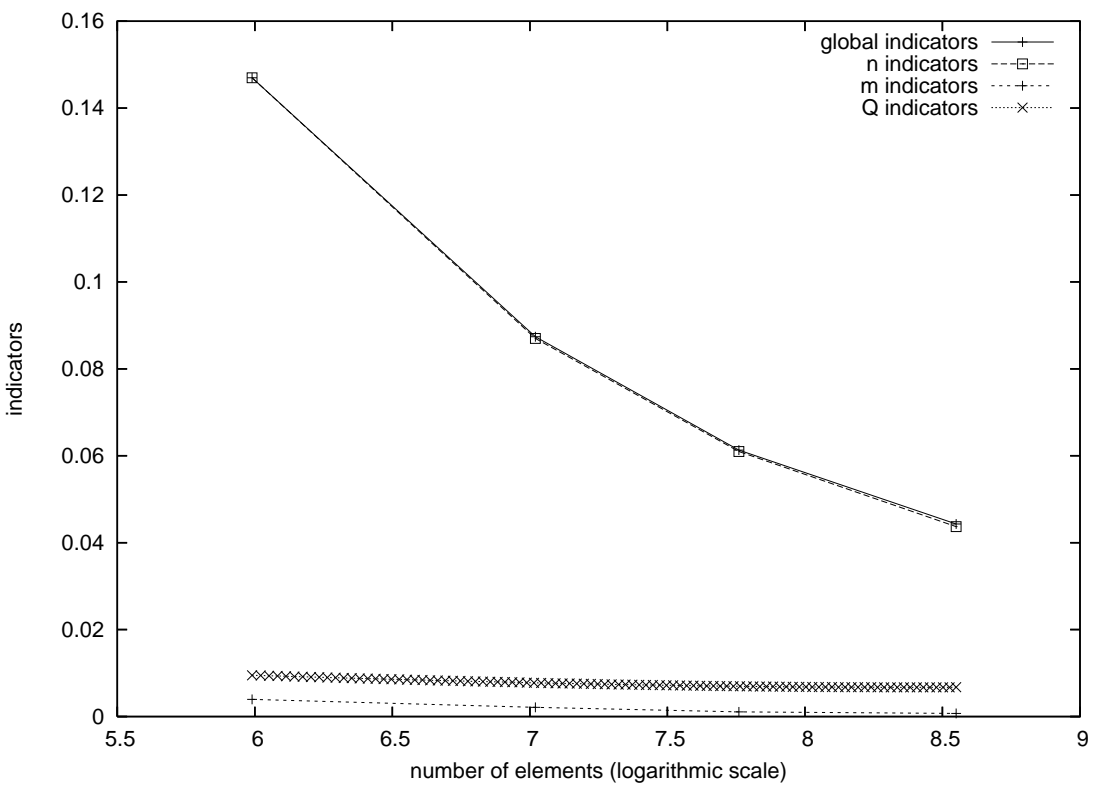

Fig. 41. Cracked cylindrical shell - Indicators $\eta, \eta_{n} / \sqrt{J_{0}}, \eta_{m} / \sqrt{J_{0}}$ and $\eta_{q} / \sqrt{J_{0}}$ near the crack tip. 


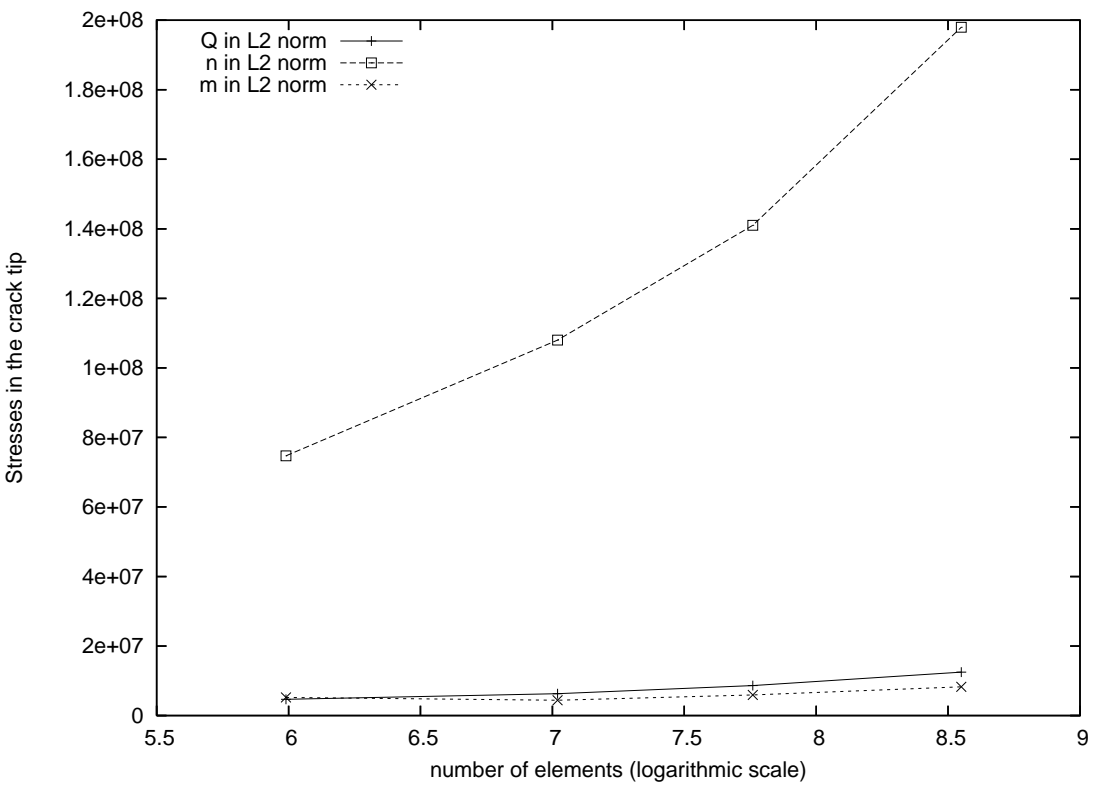

Fig. 42. Cracked cylindrical shell - $\|q\|_{L^{2}},\|n\|_{L^{2}}$ and $\|m\|_{L^{2}}$ near the crack tip. 


\begin{tabular}{|c|c|c|c|c|c|}
\hline length & thickness & radius & Young's modulus & Poisson's ratio & pressure \\
$L=100$ & $2 \varepsilon=1$ & $R=100$ & $E=3 \times 10^{9}$ & $\nu=0.3$ & $P=3 \times 10^{5}$ \\
\hline
\end{tabular}

Table 1

Settings for the cylinder under uniform pressure and the cracked cylindrical shell. 


\begin{tabular}{|c|c|c|}
\hline Young's modulus & Poisson's ratio & internal pressure \\
$E=68.25 \times 10^{6} \mathrm{~Pa}$ & $\nu=0.3$ & $P=3 \times 10^{3} \mathrm{~Pa}$ \\
\hline
\end{tabular}

Table 3

Settings for the tank under uniform pressure - Mechanical aspects. 